\title{
Correspondence
}

\section{Compressive-Sensing-Based Multiuser Detector for the 2 Large-Scale SM-MIMO Uplink}

3 Zhen Gao, Linglong Dai, Zhaocheng Wang, Senior Member, IEEE, 4 Sheng Chen, Fellow, IEEE, and Lajos Hanzo, Fellow, IEEE

5 Abstract-Conventional spatial modulation (SM) is typically consid6 ered for transmission in the downlink of small-scale multiple-input7 multiple-output (MIMO) systems, where a single antenna element (AE) of 8 a set of, e.g., $2^{p}$ AEs is activated for implicitly conveying $p$ bits. By contrast, 9 inspired by the compelling benefits of large-scale MIMO (LS-MIMO) 10 systems, here, we propose an LS-SM-MIMO scheme for the uplink (UL), 11 where each user having multiple AEs but only a single radio frequency 12 (RF) chain invokes SM for increasing the UL throughput. At the same time, 13 by relying on hundreds of AEs and a small number of $\mathbf{R F}$ chains, the base 14 station (BS) can simultaneously serve multiple users while reducing the 15 power consumption. Due to the large number of AEs of the UL users and 16 the comparably small number of RF chains at the BS, the UL multiuser sig17 nal detection becomes a challenging large-scale underdetermined problem. 18 To solve this problem, we propose a joint SM transmission scheme and 19 a carefully designed structured compressive sensing (SCS)-based multi20 user detector (MUD) to be used at the users and the BS, respectively. 21 Additionally, the cyclic-prefix single carrier (CPSC) is used to combat 22 the multipath channels, and a simple receive AE selection is used for the 23 improved performance over correlated Rayleigh-fading MIMO channels. 24 We demonstrate that the aggregate SM signal consisting of multiple UL 25 users' SM signals of a CPSC block exhibits distributed sparsity. Moreover, 26 due to the joint SM transmission scheme, aggregate SM signals in the same 27 transmission group exhibit group sparsity. By exploiting these intrinsically 28 sparse features, the proposed SCS-based MUD can reliably detect the 29 resultant SM signals with low complexity. Simulation results demonstrate 30 that the proposed SCS-based MUD achieves a better signal detection 31 performance than its counterparts even with higher UL throughput.

32 Index Terms-Compressive sensing (CS), large-scale multiple-input33 multiple-output (LS-MIMO), multiuser detector (MUD), spatial modula34 tion (SM).

\section{INTRODUCTION}

36 A widely recognized consensus is that fifth-generation (5G) sys37 tems will be capable of providing significant energy efficiency and 38 system capacity improvements [1], [2]. Promising techniques, such 39 as large-scale multiple-input-multiple-output (LS-MIMO) and spatial 40 modulation (SM)-MIMO systems, are considered as potent candidates 41 for 5G systems [1]-[5]. LS-MIMO employing hundreds of antenna

Manuscript received May 7, 2015; revised October 1, 2015; accepted November 14, 2015. This work was supported in part by the International Science and Technology Cooperation Program of China under Grant 2015DFG12760, by the National Natural Science Foundation of China under Grant 61571270 and Grant 61201185 , by the Beijing Natural Science Foundation under Grant 4142027, and by the Foundation of Shenzhen government. The review of this paper was coordinated by Dr. Y. Xin.

Z. Gao, L. Dai, and Z. Wang are with the Tsinghua National Laboratory for Information Science and Technology (TNList), Department of Electronic Engineering, Tsinghua University, Beijing 100084, China (e-mail: gao-z11@ mails. tsinghua.edu.cn; daill@mail.tsinghua.edu.cn; zcwang@mail.tsinghua.edu.cn).

S. Chen and L. Hanzo are with the School of Electronics and Computer Science, University of Southampton, Southampton SO17 1BJ, U.K. (e-mail: sqc@ecs.soton.ac.uk; lh@ecs.soton.ac.uk).

Color versions of one or more of the figures in this paper are available online at http://ieeexplore.ieee.org.

Digital Object Identifier 10.1109/TVT.2015.2501460 elements (AEs) at the base station (BS) is capable of improving 42 spectral efficiency by orders of magnitude, but it suffers from the 43 nonnegligible power consumption and hardware cost due to one spe- 44 cific radio frequency (RF) chain usually required by every AE [5]. 45 By using a reduced number of RF chains, the emerging SM-MIMO 46 activates part of available AEs to transmit extra information in the 47 spatial domain, and it has attracted much attention due to its high en- 48 ergy efficiency and reduced hardware cost [5]. However, conventional 49 SM-MIMO is usually considered in the downlink of small-scale 50 MIMO systems, and therefore, its achievable capacity is limited. Indi- 51 vidually, both technologies have their own advantages and drawbacks. 52 By an effective combination of them, one can envision the win-win 53 situation. SM-MIMO is attractive for LS-MIMO systems, since the 54 reduced number of required RF chains in SM-MIMO can reduce the 55 power consumption and hardware cost in conventional LS-MIMO 56 systems. Moveover, hundreds of AEs used in LS-MIMO can im- 57 prove the system throughput of SM-MIMO. Such reciprocity enables 58 LS-MIMO and SM-MIMO to enjoy the apparent compatibility.

In this paper, we propose an LS-SM-MIMO scheme for intrinsi- 60 cally amalgamating the compelling benefits of both LS-MIMO and 61 SM-MIMO for the 5G uplink (UL) over frequency-selective fading 62 channels. In the proposed scheme, each UL user equipped with mul- 63 tiple AEs but only a single RF chain invokes SM for increasing the 64 UL throughput, and the cyclic-prefix single-carrier (CPSC) transmis- 65 sion scheme is adopted to combat the multipath channels [6]. At the 66 BS, hundreds of AEs but only dozens of RF chains are employed to 67 simultaneously serve multiple users, and a direct AE selection scheme 68 is used to improve the system performance over correlated Rayleigh- 69 fading MIMO channels at the BS [7]. The proposed scheme can 70 be adopted in conventional LS-MIMO as a specific UL-transmission 71 mode for reducing the power consumption or, alternatively, for energy- 72 and cost-efficient LS-SM-MIMO, where joint benefits of efficient AE 73 selection [7], transmit precoding [8], and channel estimation [9] can 74 be readily exploited. To sum up, the proposed scheme inherits the 75 advantages of LS-MIMO and SM-MIMO, while reducing the power 76 consumption and hardware cost.

A challenging problem in the proposed UL LS-SM-MIMO scheme 78 is how to realize a reliable multiuser detector (MUD) with low com- 79 plexity. The optimal maximum likelihood (ML) signal detector suffers 80 from excessive complexity. Conventional sphere decoding detectors 81 cannot be readily used in multiuser scenarios and may still exhibit high 82 complexity for LS-SM-MIMO [10]. Existing low-complexity linear 83 signal detectors, e.g., the minimum mean square error (MMSE)-based 84 signal detector, perform well for conventional LS-MIMO systems 85 [4]. However, they are unsuitable for the proposed LS-SM-MIMO 86 UL transmission, since the large number of transmit AEs of the UL 87 users and the reduced number of receive RF chains at the BS make 88 UL multiuser signal detection a large-scale underdetermined/rank- 89 deficient problem. The authors in [11]-[13] proposed compressive 90 sensing (CS)-based signal detectors to solve the underdetermined 91 signal detection problem in SM-MIMO systems, but they only consid- 92 ered the single-user small-scale SM-MIMO systems in the downlink. 93

Against this background, our new contribution is that we exploit 94 the specific signal structure in the proposed multiuser LS-SM-MIMO 95 UL transmission, where each user only activates a single AE in each 96 time slot. Hence, the SM signal of each UL user is sparse with 97 
98 the sparsity level of one, and the aggregate SM signal consisting 99 of multiple UL users' SM signals of a CPSC block exhibits certain 100 distributed sparsity, which can be beneficially exploited for improving 101 the signal detection performance at the BS. Moreover, we propose 102 a joint SM transmission scheme for the UL users in conjunction 103 with an appropriately structured CS (SCS)-based MUD at the BS. 104 The proposed SCS-based MUD is specifically tailored to leverage 105 the inherently distributed sparsity of the aggregate SM signal and the 106 group sparsity of multiple aggregate SM signals, owing to the joint SM 107 transmission scheme for reliable signal detection performance. Our 108 simulation results demonstrate that the proposed SCS-based MUD is 109 capable of outperforming the conventional detectors even with higher 110 UL throughput.

111 The rest of this paper is organized as follows. Section II introduces 112 the system model of the proposed LS-SM-MIMO scheme. Section III 113 specifies the proposed joint SM transmission and SCS-based MUD. 114 Section IV provides our simulation results. Section V concludes this 115 paper.

116 Throughout this paper, lowercase and uppercase boldface letters 117 denote vectors and matrices, respectively, whereas $(\cdot)^{T},(\cdot)^{*},(\cdot)^{\dagger}$, and $118\lfloor\cdot \mid$ denote the transpose, conjugate transpose, Moore-Penrose matrix 119 inversion, and the integer floor operators, respectively. The $l_{0}$ and $120 l_{2}$ norm operations are given by $\|\cdot\|_{0}$ and $\|\cdot\|_{2}$, respectively. The 121 support set of vector $\mathbf{x}$ is denoted by $\operatorname{supp}\{\mathbf{x}\}$, and $\mathbf{x}\rangle_{i}$ denotes the $i$ th 122 entry of vector $\mathbf{x}$. Additionally, $\left.\mathbf{x}\right|_{\Gamma}$ denotes the entries of $\mathbf{x}$ defined 123 in the set $\Gamma,\left.\boldsymbol{\Phi}\right|_{\Gamma}$ denotes the submatrix whose columns comprise the 124 columns of $\boldsymbol{\Phi}$ that are defined in $\Gamma$, and $\boldsymbol{\Phi}\rangle_{\Gamma}$ denotes the submatrix 125 whose rows comprise the rows of $\boldsymbol{\Phi}$ that are defined in $\Gamma$. The 126 expectation operator is given by $E\{\cdot\} . \bmod (x, y)=x-\lfloor x / y\rfloor y$ if $127 y \neq 0$ and $x-\lfloor x / y\rfloor y \neq 0$, whereas $\bmod (x, y)=y$ if $y \neq 0$ and $128 x-\lfloor x / y\rfloor y=0$.

\section{SYSTEM MODEL}

130 We first introduce the proposed LS-SM-MIMO scheme and then 131 focus our attention on the UL transmission with an emphasis on the 132 multiuser signal detection.

\section{A. Proposed Multiuser LS-SM-MIMO Scheme}

134 As shown in Fig. 1, we consider the proposed LS-SM-MIMO from 135 both the BS side and the user side. For conventional LS-MIMO, the 136 number of AEs employed by the BS is equal to the number of its RF 137 chains [4]. However, the BS in LS-SM-MIMO, as shown in Fig. 1, 138 is equipped with a much smaller number of RF chains $M_{\mathrm{RF}}$ than 139 the total number of AEs $M$, i.e., we have $M_{\mathrm{RF}} \ll M$. Conventional 140 LS-MIMO systems typically assume single-antenna users [4]. By 141 contrast, in the proposed scheme, each user is equipped with $n_{t}>1$ 142 AEs but only a single RF chain, and SM is adopted for the UL 143 transmission, where only one of the available AEs is activated for 144 data transmission. It has been shown that the main power consumption 145 and hardware cost of cellular networks comes from the radio access 146 network [1]. Hence, using a reduced number of expensive RF chains 147 compared with the total number of AEs at the BS can substantially 148 reduce both the power consumption and the hardware cost for the 149 operators. Meanwhile, it is feasible to incorporate several AEs and a 150 single RF chain in the handsets. The resultant increased degrees of 151 freedom in the spatial domain may then be exploited for improving 152 the UL throughput. The proposed scheme can be considered as an 153 optional UL-transmission mode in conventional LS-MIMO systems, 154 where AE selection schemes may be adopted for beneficially selecting 155 the most suitable $M_{\mathrm{RF}}$ AEs at the BS to receive UL SM signals [7]. 156 Alternatively, it can also be used for the UL of LS-SM-MIMO, when

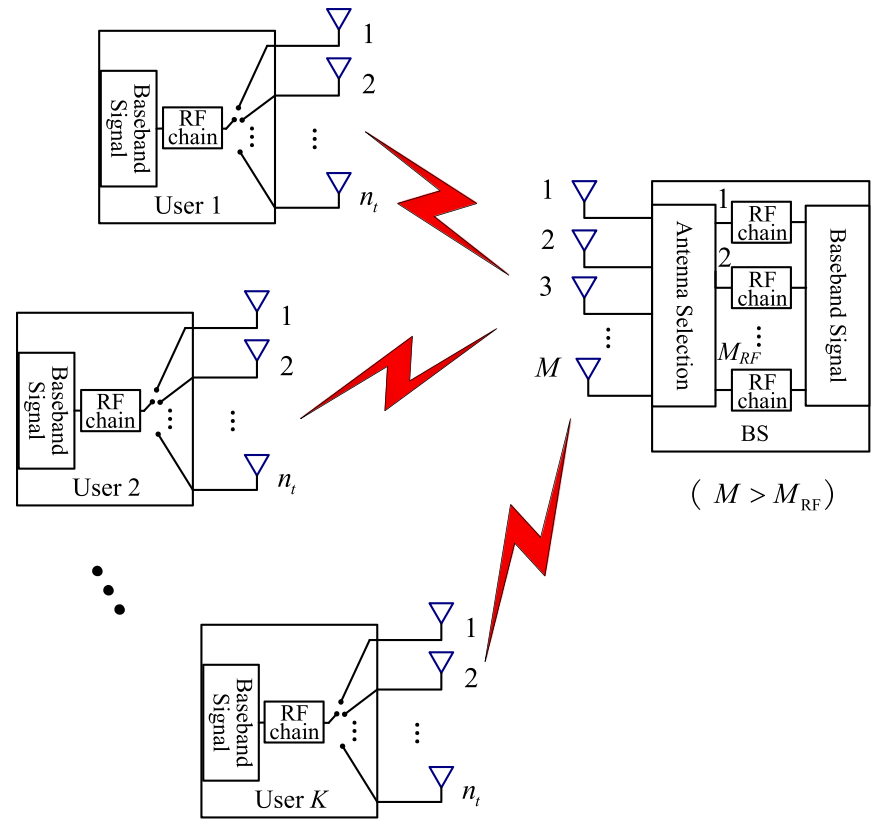

Fig. 1. In the proposed UL LS-SM-MIMO, the BS is equipped with $M$ AEs and $M_{\mathrm{RF}} \mathrm{RF}$ chains to simultaneously serve $K$ users, where $M \gg M_{\mathrm{RF}}>$ $K$, and each user is equipped with $n_{t}>1$ AEs and one RF chain. By exploiting the improved degree of freedom in the spatial domain, multiple users can simultaneously exploit SM for improving the UL throughput.

advantageously combining transmit precoding, receive AE selection, 157 and channel estimation [7]-[9].

\section{B. Uplink Multiuser Transmission}

We first consider the generation of SM signals at the users. The SM 160 signal $\mathbf{x}_{k}=\mathbf{e}_{k} s_{k}$ transmitted by the $k$ th user in a time slot consists 161 of two parts: the spatial constellation symbol $\mathbf{e}_{k} \in \mathbb{C}^{n_{t}}$ and the signal 162 constellation symbol $s_{k} \in \mathbb{C}$. $\mathbf{e}_{k}$ is generated by mapping $\left\lfloor\log _{2}\left(n_{t}\right)\right\rfloor 163$ bits to the index of the active AE, and typically, the user terminal 164 employs $n_{t}=2^{p}$ AEs, where $p$ is a positive integer. Due to only a 165 single RF chain employed at each user, only one entry of $\mathbf{e}_{k}$ associated 166 with the active $\mathrm{AE}$ is equal to one, and the rest of the entries of $\mathbf{e}_{k}$ are 167 zeros, i.e., we have

$$
\operatorname{supp}\left(\mathbf{e}_{k}\right) \in \mathbb{A}, \quad\left\|\mathbf{e}_{k}\right\|_{0}=1, \quad\left\|\mathbf{e}_{k}\right\|_{2}=1
$$

where $\mathbb{A}=\left\{1,2, \ldots, n_{t}\right\}$ is the spatial constellation symbol set. The 169 signal constellation symbol comes from $L$-ary modulation, i.e., $s_{k} \in 170$ $\mathbb{L}$, where $\mathbb{L}$ is the signal constellation symbol set [e.g., 64 quadrature 171 amplitude modulation (QAM)] of size $L$. Hence, each UL user's SM 172 signal carries the information of $\log _{2}(L)+\log _{2}\left(n_{t}\right)$ bits per channel 173 use (bpcu), and the overall UL throughput is $K\left(\log _{2}(L)+\log _{2}\left(n_{t}\right)\right) 174$ bpcu. The users rely on the CPSC scheme for transmitting their SM 175 signals [6]. Explicitly, each CPSC block consists of a cyclic prefix 176 (CP) having the length of $P-1$ and the associated data block having 177 the length of $Q$. Hence, the length of each CPSC block is $Q+P-1,178$ where this $\mathrm{CP}$ is capable of counteracting a dispersive multipath 179 channel imposing dispersion over $P$ samples. The concatenated data 180 block consists of $Q$ successive SM signals.

At the receiver, due to the reduced number of RF chains at the BS, 182 only $M_{\mathrm{RF}}$ receive AEs can be exploited to receive signals, where exist- 183 ing receive AE selection schemes can be adopted to preselect $M_{\mathrm{RF}}$ re- 184 ceive AEs for achieving an improved signal detection performance [7]. 185 Since the BS can serve $K$ users simultaneously, after the removal of 186 
187 the $\mathrm{CP}$, the received signal $\mathbf{y}_{q} \in \mathbb{C}^{M_{\mathrm{RF}}}$ for $1 \leq q \leq Q$ of the $q$ th time 188 slot of a specific CPSC block can be expressed as

$$
\begin{aligned}
\mathbf{y}_{q}=\sum_{k=1}^{K} \mathbf{y}_{k, q}+\mathbf{w}_{q} & \left.=\sum_{p=0}^{P-1} \sum_{k=1}^{K} \mathbf{H}_{k, p}\right\rangle_{\Theta} \mathbf{x}_{k,} \bmod (q-p, Q) \\
& +\mathbf{w}_{q}=\sum_{p=0}^{P-1} \sum_{k=1}^{K} \tilde{\mathbf{H}}_{k, p} \mathbf{x}_{k,} \bmod (q-p, Q)+\mathbf{w}_{q}
\end{aligned}
$$

189 where $\mathbf{H}_{k, p} \in \mathbb{C}^{M \times n_{t}}$ is the $k$ th user's MIMO channel matrix for the $190 p$ th multipath component, i.e., $\left.\mathbf{H}_{k, p}\right\rangle_{\Theta}=\tilde{\boldsymbol{H}}_{k, p} \in \mathbb{C}^{M_{\mathrm{RF}} \times n_{t}}$; the set $191 \Theta$ is determined by the AE selection scheme used; the elements of $192 \Theta$ having the cardinality of $M_{\mathrm{RF}}$ are uniquely selected from the set $193\{1,2, \ldots, M\} ; \mathbf{x}_{k, q}$ has one nonzero entry; and $\mathbf{w}_{q} \in \mathbb{C}^{M_{\mathrm{RF}}}$ is the 194 additive white Gaussian noise (AWGN) vector with entries obeying 195 the independent and identically distributed (i.i.d.) circularly symmet196 ric complex Gaussian distribution with zero mean and a variance 197 of $\sigma_{w}^{2} / 2$ per dimension, which is denoted by $\mathcal{C N}\left(0, \sigma_{w}^{2}\right) . \mathbf{H}_{k, p}=$ $198 \mathbf{R}_{\mathrm{BS}}^{1 / 2} \overline{\boldsymbol{H}}_{k, p} \mathbf{R}_{\mathrm{US}}^{1 / 2}$, the entries of $\overline{\boldsymbol{H}}_{k, p}$ obey the i.i.d. $\mathcal{C N}(0,1)$, and $199 \mathbf{R}_{\mathrm{US}}$ with the correlation coefficient $\rho_{\mathrm{US}}$ and $\mathbf{R}_{\mathrm{BS}}$ with the correlation 200 coefficient $\rho_{\mathrm{BS}}$ are correlation matrices at the users and the BS, 201 respectively. The specific element of the $m$ th row and the $n$th column 202 of $R_{\mathrm{BS}}\left(R_{\mathrm{US}}\right)$ is $\rho_{\mathrm{BS}}^{|m-n|}\left(\rho_{\mathrm{US}}^{|m-n|}\right)$. For correlated Rayleigh-fading 203 MIMO channels, the specific $\Theta$ or receive AE selection scheme has 204 an impact on the attainable system performance. In this paper, the 205 direct $\mathrm{AE}$ selection scheme is used for maximizing the minimum 206 geometric distance between any pair of the selected AEs [7]. For 207 uniform linear arrays (ULAs), $\Theta=\left\{\varphi+m_{\mathrm{RF}}\left\lfloor M / M_{\mathrm{RF}}\right\rfloor\right\}_{m_{\mathrm{RF}}=0}^{M_{\mathrm{RF}}-1}$ 208 with $1 \leq \varphi \leq\left\lfloor M / M_{\mathrm{RF}}\right\rfloor-1$. Then, (2) can be further expressed as

$$
\mathbf{y}_{q}=\sum_{p=0}^{P-1} \tilde{\mathbf{H}}_{p} \mathbf{x} \bmod (q-p, Q)+\mathbf{w}_{q}
$$

209 by defining $\tilde{\boldsymbol{H}}_{p}=\left[\tilde{\boldsymbol{H}}_{1, p} \tilde{\boldsymbol{H}}_{2, p}, \ldots, \tilde{\boldsymbol{H}}_{K, p}\right] \in \mathbb{C}^{M_{\mathrm{RF}} \times\left(n_{t} K\right)}$ and $210 \mathbf{x}_{q}=\left[\left(\mathbf{x}_{1, q}\right)^{T}\left(\mathbf{x}_{2, q}\right)^{T}, \ldots,\left(\mathbf{x}_{K, q}\right)^{T}\right]^{T} \in \mathbb{C}^{\left(n_{t} K\right)}$. By considering 211 the $Q$ SM signals of a specific CPSC block, we arrive at

$$
\mathbf{y}=\tilde{\mathbf{H}} \mathbf{x}+\mathbf{w}
$$

212 where $\mathbf{y}=\left[\left(\mathbf{y}_{1}\right)^{T}\left(\mathbf{y}_{2}\right)^{T}, \ldots,\left(\mathbf{y}_{Q}\right)^{T}\right]^{T} \in^{\left(M_{\mathrm{RF}} Q\right)}$, the aggregate $213 \mathrm{SM}$ signal $\mathbf{x}=\left[\left(\mathbf{x}_{1}\right)^{T}\left(\mathbf{x}_{2}\right)^{T}, \ldots,\left(\mathbf{x}_{Q}\right)^{T}\right]^{T} \in \in^{\left(K n_{t} Q\right)}, \quad \mathbf{w}=$ $214\left[\left(\mathbf{w}_{1}\right)^{T}\left(\mathbf{w}_{2}\right)^{T}, \ldots,\left(\mathbf{w}_{Q}\right)^{T}\right]^{T}$, and

$\tilde{\mathbf{H}}=\left[\begin{array}{cccccc}\tilde{\mathbf{H}}_{0} & \mathbf{0} & \mathbf{0} & \ldots & \tilde{\mathbf{H}}_{2} & \tilde{\mathbf{H}}_{1} \\ \tilde{\mathbf{H}}_{1} & \tilde{\mathbf{H}}_{0} & \mathbf{0} & \ldots & \vdots & \tilde{\mathbf{H}}_{2} \\ \vdots & \tilde{\mathbf{H}}_{1} & \tilde{\mathbf{H}}_{0} & \ldots & \tilde{\mathbf{H}}_{P-1} & \vdots \\ \tilde{\mathbf{H}}_{P-1} & \vdots & \tilde{\mathbf{H}}_{1} & \ldots & \mathbf{0} & \tilde{\mathbf{H}}_{P-1} \\ \mathbf{0} & \tilde{\mathbf{H}}_{P-1} & \vdots & \vdots & \vdots & \mathbf{0} \\ \vdots & \mathbf{0} & \tilde{\mathbf{H}}_{P-1} & \vdots & \vdots & \vdots \\ \vdots & \vdots & \mathbf{0} & \vdots & \mathbf{0} & \vdots \\ \vdots & \vdots & \vdots & \vdots & \tilde{\mathbf{H}}_{0} & \mathbf{0} \\ \mathbf{0} & \mathbf{0} & \mathbf{0} & \cdots & \tilde{\mathbf{H}}_{1} & \tilde{\mathbf{H}}_{0}\end{array}\right]$.

215 The signal-to-noise ratio (SNR) at the receiver is defined by SNR = $216 E\left\{\|\tilde{\boldsymbol{H}} \mathbf{x}\|_{2}^{2}\right\} / E\left\{\|\mathbf{w}\|_{2}^{2}\right\}$.
The optimal signal detector for (4) relies on the ML algorithm, i.e., 217

$$
\begin{aligned}
\min _{\hat{\mathbf{x}}} & \|\mathbf{y}-\tilde{\mathbf{H}} \hat{\mathbf{x}}\|_{2}=\min _{\left\{\hat{\mathbf{x}}_{k, q}\right\}_{k=1, q=1}^{K, Q}}\|\mathbf{y}-\tilde{\mathbf{H}} \hat{\mathbf{x}}\|_{2} \\
\text { s.t. } & \left.\operatorname{supp}\left(\hat{\mathbf{x}}_{k, q}\right) \in \mathbb{A}, \hat{\mathbf{x}}_{k, q}\right\rangle_{\operatorname{supp}\left(\hat{\mathbf{x}}_{k, q}\right)} \in \mathbb{L}, 1 \leq k \leq K, 1 \leq q \leq Q
\end{aligned}
$$

whose complexity exponentially increases with the number of users, 218 since the size of the search set for the ML detector is $\left(n_{t} \cdot L\right)^{K Q} .219$ This excessive complexity can be unaffordable in practice. To reduce 220 the complexity, near-optimal sphere decoding detectors have been 221 proposed [10], but their complexity may still remain high, particularly 222 for the systems supporting large $K, Q, n_{t}$, and $L$ [11]. In conventional 223 LS-MIMO systems, low-complexity linear signal detectors (e.g., the 224 MMSE-based signal detector) have been shown to be near optimal 225 since $M=M_{\mathrm{RF}} \gg K$ and $n_{t}=1$ make multiuser signal detection 226 an overdetermined problem [4]. However, in the proposed scheme, we 227 have $M_{\mathrm{RF}}<K n_{t}$. Hence, the multiuser signal detection problem (6) 228 represents a large-scale underdetermined problem. Consequently, the 229 conventional linear signal detectors perform poorly in the proposed 230 LS-SM-MIMO [11]. By exploiting the sparsity of SM signals, the 231 authors in [11]-[13] have proposed the concept of CS-based signal 232 detectors for the downlink of small-scale SM-MIMO operating in a 233 single-user scenario. However, these signal detectors are unsuitable 234 for the proposed multiuser scenarios. Observe from (1) that $\mathbf{x}_{k, q}$ is 235 a sparse signal having a sparsity level of one. Hence, the aggregate 236 SM signal $\mathbf{x}$, which consists of multiple users' SM signals in $Q 237$ time slots, exhibits distributed sparsity with the sparsity level of $K Q .238$ This property of $\mathbf{x}$ inspires us to exploit the SCS theory for the 239 multiuser signal detection [14]. To further improve the signal detection 240 performance and to increase the system's throughput, we propose a 241 joint SM transmission scheme and an SCS-based MUD, which will be 242 detailed in the following section.

\section{SCS-BASED MUD FOR LS-SM-MIMO UL}

To solve the multiuser signal detection of our UL LS-SM-MIMO 245 system, we first propose a joint SM transmission scheme to be 246 employed at the users. Accordingly, an SCS-based low-complexity 247 MUD is developed at the BS, whereby the distributed sparsity of the 248 aggregate SM signal and the group sparsity of multiple aggregate SM 249 signals are exploited. Moreover, the computational complexity of the 250 proposed SCS-based MUD is discussed.

\section{A. Joint SM Transmission Scheme at the Users}

For the $k$ th user in the $q$ th time slot, every successive $J$ CPSC block 253 is considered as a group, and they share the same spatial constellation 254 symbol, i.e.,

$$
\begin{aligned}
\operatorname{supp}\left(\mathbf{x}_{k, q}^{(1)}\right)=\operatorname{supp}\left(\mathbf{x}_{k, q}^{(2)}\right)=\cdots= & \operatorname{supp}\left(\mathbf{x}_{k, q}^{(J)}\right), \\
& 1 \leq k \leq K, 1 \leq q \leq Q
\end{aligned}
$$

where we introduce the superscript $(j)$ to denote the $j$ th CPSC block, 256 and $J$ is typically small, e.g., $J=2$. In CS theory, the specific signal 257 structure, where $\mathbf{x}_{k, q}^{(1)}, \mathbf{x}_{k, q}^{(2)}, \ldots, \mathbf{x}_{k, q}^{(J)}$ share a common support, is often 258 referred to as the group sparsity. Similarly, the aggregate SM signals 259 consisting of the $K$ users' SM signals also exhibit group sparsity, i.e., 260

$$
\operatorname{supp}\left(\mathbf{x}^{(1)}\right)=\operatorname{supp}\left(\mathbf{x}^{(2)}\right)=\cdots=\operatorname{supp}\left(\mathbf{x}^{(J)}\right) .
$$

Although exhibiting group sparsity may slightly reduce the informa- 261 tion carried by the spatial constellation symbols, it is also capable of 262 
263 reducing the number of the RF chains required according to the SCS 264 theory, while simultaneously improving the total bit error rate (BER) 265 of the entire system even with higher UL throughput. This conclusion 266 will be confirmed by our simulation results.

\section{B. SCS-Based MUD at the BS}

268 According to (4), the received signals at the BS in the same group 269 can be expressed as

$$
\mathbf{y}^{(j)}=\tilde{\mathbf{H}}^{(j)} \mathbf{x}^{(j)}+\mathbf{w}^{(j)}, \quad 1 \leq j \leq J
$$

270 where $\mathbf{y}^{(j)}$ denotes the received signal in the $j$ th CPSC block, whereas $271 \tilde{\boldsymbol{H}}^{(j)}$ and $\mathbf{w}^{(j)}$ are the effective MIMO channel matrix and the AWGN 272 vector, respectively.

273 The intrinsically distributed sparsity of $\mathbf{x}^{(j)}$ and the underdeter274 mined nature of (9) inspire us to solve the signal detection problem 275 based on CS theory, which can efficiently acquire the sparse solutions 276 to underdetermined linear systems. Moreover, the $J$ different aggre277 gate SM signals in (9) can be jointly exploited for improving the signal 278 detection performance due to the group sparsity of $\left\{\mathbf{x}^{(j)}\right\}_{j=1}^{J}$. Thus, 279 by considering both the distributed sparsity and the group sparsity of 280 the aggregate SM signals, the multiuser signal detection at the BS can 281 be formulated as the following optimization problem:

$$
\begin{array}{cl}
\underset{\left\{\widehat{\mathbf{x}}^{(j)}\right\}_{j=1}^{J}}{\min } & \sum_{j=1}^{J}\left\|\mathbf{y}^{(j)}-\tilde{\mathbf{H}}^{(j)} \widehat{\mathbf{x}}^{(j)}\right\|_{2}^{2}={\underset{\left\{\widehat{\mathbf{x}}_{k, q}^{(j)}\right\}_{j=1, k=1, q=1}^{J, K, Q}}{j=1, k}} \\
& \sum_{j=1}^{J}\left\|\mathbf{y}^{(j)}-\tilde{\mathbf{H}}^{(j)} \widehat{\mathbf{x}}^{(j)}\right\|_{2}^{2} \\
\text { s.t. } & \left\|\widehat{\mathbf{x}}_{k, q}^{(j)}\right\|_{0}=1,1 \leq j \leq J, 1 \leq q \leq Q, 1 \leq k \leq K .
\end{array}
$$

282 Our proposed SCS-based MUD solves the optimization problem (10) 283 with the aid of two steps. In the first step, we estimate the spatial 284 constellation symbols, i.e., the indexes of $K$ users' active AEs in $J$ 285 successive CPSC blocks. In the second step, we infer the legitimate 286 signal constellation symbols of the $K$ users in $J$ CPSC blocks.

287 1. Step 1-Estimation of Spatial Constellation Symbols: We pro288 pose a group subspace pursuit (GSP) algorithm developed from the 289 classical subspace pursuit (SP) algorithm in [15] to acquire the 290 sparse solution to the large-scale underdetermined problem (10), 291 where both the a priori sparse information (i.e., $\left\|\mathbf{x}_{k, q}^{(j)}\right\|_{0}=1$ ) and 292 the group sparsity of $\mathbf{x}^{(1)}, \mathbf{x}^{(2)}, \ldots, \mathbf{x}^{(J)}$ are exploited for improv293 ing the multiuser signal detection performance. The proposed GSP 294 algorithm is described in Algorithm 1, which estimates SM signal $295\left\{\widehat{\mathbf{x}}_{k, q}^{(j)}\right\}_{k=1, j=1, q=1}^{K, J, Q}$. Hence, the estimated spatial constellation symbol 296 is $\left\{\operatorname{supp}\left(\widehat{\mathbf{x}}_{k, q}^{(j)}\right)\right\}_{k=1, j=1, q=1}^{K, J, Q}$.

\section{Algorithm 1 Proposed GSP Algorithm.}

Input: Noisy received signals $\mathbf{y}^{(j)}$ and effective channel matrices $\tilde{\boldsymbol{H}}^{(j)}$ for $1 \leq j \leq J$. Output:Estimated $\widehat{\mathbf{x}}^{(j)}=\left[\left(\widehat{\mathbf{x}}_{1}^{(j)}\right)^{T}\left(\widehat{\mathbf{x}}_{2}^{(j)}\right)^{T}, \ldots,\left(\widehat{\mathbf{x}}_{Q}^{(j)}\right)^{T}\right]^{T}$, where $\widehat{\mathbf{x}}_{q}^{(j)}=\left[\left(\widehat{\mathbf{x}}_{1, q}^{(j)}\right)^{T}\left(\widehat{\mathbf{x}}_{2, q}^{(j)}\right)^{T}, \ldots,\left(\widehat{\mathbf{x}}_{K, q}^{(j)}\right)^{T}\right]^{T}$ for $1 \leq q \leq Q$. $1: \mathbf{r}^{(j)}=\mathbf{y}^{(j)}$ for $1 \leq j \leq J ;\{$ Initialization $\}$ 2: $\Omega^{0}=\emptyset ;\{$ Empty support set $\}$

$303 \quad 3: t=1 ;\{$ Iteration index $\}$
5: $\quad \mathbf{a}_{k, q}^{(j)}=\left(\tilde{\boldsymbol{H}}_{k, q}^{(j)}\right)^{*} \mathbf{r}^{(j)}$ for $1 \leq k \leq K, 1 \leq q \leq Q$, and $1 \leq j \leq J ; 305$ \{Correlation\}

6: $\left.\quad \tau_{k, q}=\arg \max _{\tau_{k, q}} \sum_{j=1}^{J} \| \mathbf{a}_{k, q}^{(j)}\right\rangle_{\tau_{k, q}} t \|_{2}^{2} \quad$ for $\quad 1 \leq k \leq K, 307$ $1 \leq q \leq Q ;$ Identify support $\}$ 308

7: $\quad \Gamma=\left\{\tau_{k, q}+(k-1+K(q-1)) n_{t}\right\}_{k=1, q=1}^{K, Q} ; \quad$ \{Preliminary 309 support set $\}$

8: $\left.\quad \mathbf{b}^{(j)}\right|_{\Omega^{t-1} \cup \Gamma}=\left(\left.\tilde{\boldsymbol{H}}^{(j)}\right|_{\Omega^{t-1} \cup \Gamma}\right)^{\dagger} \mathbf{y}^{(j)}$ for $1 \leq j \leq J ;$ Least 311 squares

9: $\left.\quad \omega_{k, q}=\arg \max _{\widetilde{\omega}_{k, q}} \sum_{j=1}^{J} \| \mathbf{b}_{k, q}^{(j)}\right\rangle_{\widetilde{\omega}_{k, q}} \|_{2}^{2} \quad$ for $\quad 1 \leq k \leq K, 313$ $1 \leq q \leq Q ;$ Pruning support set $\}$

10: $\quad \bar{\Omega}^{t}=\left\{\omega_{k, q}+(k-1+K(q-1)) n_{t}\right\}_{k=1, q=1}^{K, Q} ; \quad$ [Final 315 support set $\}$

11: $\left.\quad \mathbf{c}^{(j)}\right|_{\Omega^{t}}=\left(\left.\tilde{\boldsymbol{H}}^{(j)}\right|_{\Omega^{t}}\right)^{\dagger} \mathbf{y}^{(j)}$ for $1 \leq j \leq J$; Least squares $\} \quad 317$

12: $\quad \mathbf{r}^{(j)}=\mathbf{y}^{(j)}-\tilde{\boldsymbol{H}}^{(j)} \mathbf{c}^{(j)}$ for $1 \leq j \leq J ;\{$ Compute residual $\} 318$

13: $t=t+1 ;\{$ Update iteration index $\quad 319$

14: until $\Omega^{t}=\Omega^{t-1}$ or $t \geq Q$

Compared with the classical SP algorithm, the proposed GSP algo- 321 rithm exploits the distributed sparsity and group sparsity of $\left\{\mathbf{x}^{(j)}\right\}_{j=1}^{J} \cdot 322$ More explicitly, $\mathbf{x}^{(j)} \in \mathbb{C}^{\left(K Q n_{t}\right)}$ consists of the $K Q$ low-dimensional 323 sparse vectors $\mathbf{x}_{k, q}^{(j)} \in \mathbb{C}^{n_{t}}$, where each $\mathbf{x}_{k, q}^{(j)}$ has the known sparsity 324 level of one, and the aggregate SM signals $\mathbf{x}^{(1)}, \mathbf{x}^{(2)}, \ldots, \mathbf{x}^{(J)}$ exhibit 325 group sparsity. Specifically, the differences between the proposed GSP 326 algorithm and the classical SP algorithm lie in the following two 327 aspects: 1) the identification of the support set including the steps 328 of the preliminary support set and the final support set as shown in 329 Algorithm 1; and 2) the joint processing of $\mathbf{y}^{(1)}, \mathbf{y}^{(2)}, \ldots, \mathbf{y}^{(J)}$. First, 330 for the support selection, taking the step of the preliminary support set 331 for instance, when selecting the preliminary support set, the classical 332 SP algorithm selects the support set associated with the first $K Q 333$ largest values of the global correlation result $\left(\tilde{\boldsymbol{H}}^{(j)}\right)^{*} \mathbf{r}^{(j)}$. By contrast, 334 the proposed GSP algorithm selects the support set associated with 335 the largest value from the local correlation result in each $\left(\tilde{\boldsymbol{H}}_{k, q}^{(j)}\right)^{*} \mathbf{r}^{(j)} .336$ This way, the distributed sparsity of the aggregate SM signal can be ex- 337 ploited for improved signal detection performance. Second, compared 338 with the classical SP algorithm, the proposed GSP algorithm jointly 339 exploits the $J$ correlated signals having the group sparsity, which can 340 bring the further improved signal detection performance.

It should be noted that even for the special case of $J=1$, i.e., 342 without using the joint SM transmission scheme, the proposed GSP 343 algorithm still achieves a better signal detection performance than 344 the classical SP algorithm when handling the aggregate SM signal, 345 since the inherently distributed sparsity of the aggregate SM signal is 346 leveraged to improve the signal detection performance.

2. Step 2-Acquisition of Signal Constellation Symbols: Following 348 Step 1, we can also acquire a rough estimate of the signal constellation 349 symbol for each user in each time slot. By searching for the minimum 350 Euclidean distance between this rough estimate of the signal constel- 351 lation symbol and the legitimate constellation symbols of $\mathbb{L}$, we can 352 obtain the final estimate of signal constellation symbols.

\section{Computational Complexity}

The optimal ML signal detector has a prohibitively high com- 355 putational complexity of $\mathcal{O}\left(\left(L \cdot n_{t}\right)^{(K \cdot Q)}\right)$ according to (6). The 356 sphere decoding detectors [10] are indeed capable of reducing the 357 computational complexity, but they may still suffer from unaffordable 358 complexity, particularly for large $K, Q, L$, and $n_{t}$ values. By contrast, 359 the conventional MMSE-based detector for LS-MIMO and CS-based 360 


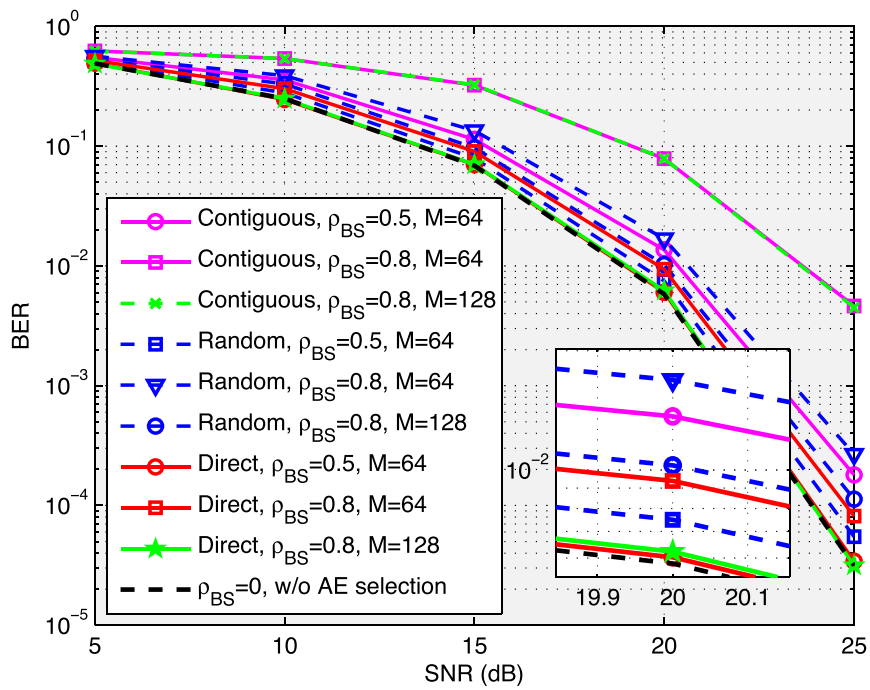

Fig. 2. Total BERs achieved by the proposed SCS-based MUD with different AE selection schemes, where $K=8, J=2,64$-QAM, $M_{\mathrm{RF}}=18, n_{t}=4$, and $\rho_{\mathrm{US}}=0$ are considered.

361 detector [13] for small-scale SM-MIMO enjoy the low complexity of $362 \mathcal{O}\left(M_{\mathrm{RF}} \cdot\left(n_{t} \cdot Q \cdot K\right)^{2}+\left(n_{t} \cdot Q \cdot K\right)^{3}\right)$ and $\mathcal{O}\left(2 M_{\mathrm{RF}} \cdot(Q \cdot K)^{2}+\right.$ $\left.363(Q \cdot K)^{3}\right)$, respectively. For the proposed SCS-based MUD, most 364 of the computational requirements are imposed by the least squares 365 (LS) operations, which has complexity of $\mathcal{O}\left(J \cdot\left(2 M_{\mathrm{RF}} \cdot(Q \cdot K)^{2}+\right.\right.$ $\left.366(Q \cdot K)^{3}\right)$ ) [16]. Consequently, the computational complexity per 367 CPSC block is $\mathcal{O}\left(2 M_{\mathrm{RF}} \cdot(Q \cdot K)^{2}+(Q \cdot K)^{3}\right)$, since $J$ successive 368 aggregate SM signals are jointly processed. Compared with conven369 tional signal detectors, the proposed SCS-based MUD benefits from 370 substantially lower complexity, and it has similar low complexity as 371 the conventional MMSE- and CS-based signal detectors.

\section{Simulation Results}

373 A simulation study was carried out to compare the attainable perfor374 mance of the proposed SCS-based MUD to that of the MMSE-based 375 signal detector [4] and to that of the CS-based signal detector [13]. 376 In the LS-SM-MIMO system considered, the BS used a ULA relying 377 on a large number of AEs $M$, but a much smaller number of RF 378 chains $M_{\mathrm{RF}}$, whereas $K$ users employing $n_{t}$ AEs but only a single RF 379 chain simultaneously use the CPSC scheme associated with $P=8$ and $380 Q=64$ to transmit the SM signals to the BS. The total BER including 381 both the spatial constellation symbols and the signal constellation 382 symbols was evaluated.

383 Fig. 2 compares the total BERs achieved by the proposed SCS-based 384 MUD with different AE selection schemes, where $K=8, J=2$, $38564-\mathrm{QAM}, M_{\mathrm{RF}}=18, n_{t}=4$, and $\rho_{\mathrm{US}}=0$ are considered. The con386 tiguous AE selection scheme implies that we select $M_{\mathrm{RF}}$ adjacent 387 AEs, i.e., $\Theta=\left\{\varphi+m_{\mathrm{RF}}\right\}_{m_{\mathrm{RF}}=0}^{M_{\mathrm{RF}}-1}$ with $1 \leq \varphi \leq M-M_{\mathrm{RF}}+1$. By 388 contrast, in the random $\mathrm{AE}$ selection scheme, the elements of $\Theta$ are 389 randomly selected from the set $\{1,2, \ldots, M\}$, whereas the direct 390 AE selection scheme in [7] has been described in Section II-B. 391 Furthermore, the BER achieved by the SCS-based MUD relying on $392 \rho_{\mathrm{BS}}=0$ is also considered as a performance bound, since the choice 393 of $\rho_{\mathrm{BS}}=0$ and $\rho_{\mathrm{US}}=0$ implies the uncorrelated Rayleigh-fading 394 MIMO channels. Observe from Fig. 2 that the direct AE selection 395 scheme outperforms the other pair of AE selection schemes. Moreover, 396 for a certain AE selection scheme, the BER performance degrades 397 when $M_{\mathrm{RF}} / M$ or $\rho_{\mathrm{BS}}$ increases. For the direct AE selection scheme, 398 the BER performance of $\rho_{\mathrm{BS}}=0.8, M=128$ and of $\rho_{\mathrm{BS}}=0.5$,

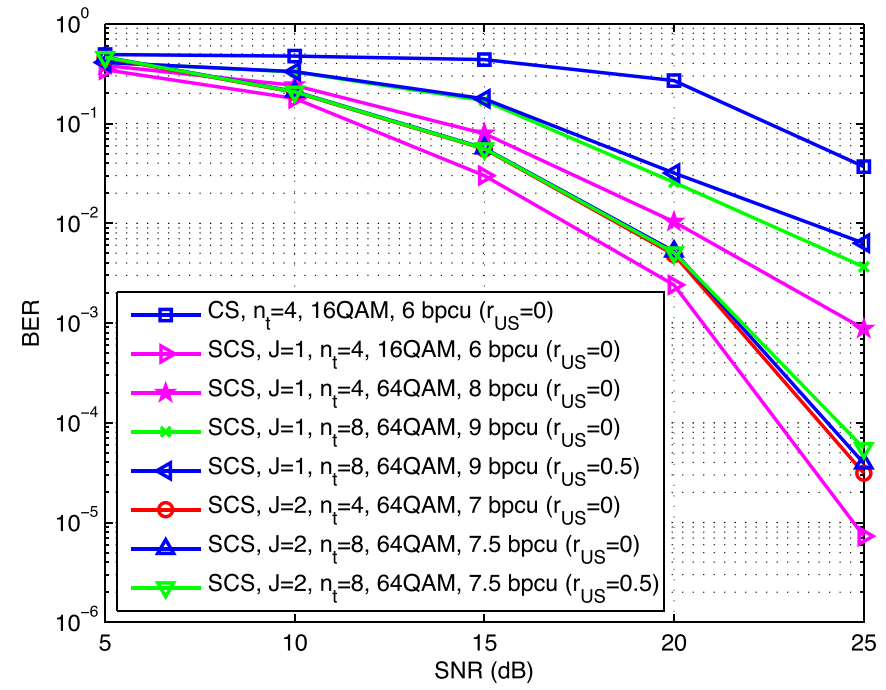

Fig. 3. Total BERs achieved by the CS-based signal detector and the SCS-based MUD against different SNRs in LS-SM-MIMO, where $K=8, M_{\mathrm{RF}}=18$, $M=64, \rho_{\mathrm{BS}}=0.5$, and the direct AE selection scheme is considered.

$M=64$ approaches the BER achieved for transmission over uncor- 399 related Rayleigh-fading MIMO channels, which confirms the near- 400 optimal performance of the direct AE selection scheme.

401

Fig. 3 compares the overall BER achieved by the CS-based signal 402 detector and by the proposed SCS-based MUD versus the SNR in our 403 LS-SM-MIMO context, where $K=8, M_{\mathrm{RF}}=18, M=64, \rho_{\mathrm{BS}}=404$ 0.5 , and the direct AE selection scheme is considered. The SCS-based 405 MUD outperforms the CS-based signal detector even for $J=1$, since 406 the distributed sparsity of the aggregate SM signal is exploited. For the 407 SCS-based MUD, the BER performance improves when $J$ increases, 408 albeit this is achieved at the cost of reduced UL throughput. To mitigate 409 this impediment, a higher number of AEs can be employed by the users 410 for expanding the spatial constellation symbol set constituted by the 411 AEs. Specifically, by increasing $n_{t}$ from 4 to 8 , the UL throughput 412 of the SCS-based MUD may be increased, but having more AEs at 413 the user results in a higher $\rho_{\text {US }}$. When $n_{t}$ is increased, the BER 414 performance of the SCS-based MUD associated with $J=1$ degrades, 415 as expected. By contrast, when $n_{t}$ is increased, the BER performance 416 loss of the SCS-based MUD using $J=2$ can be less than $0.2 \mathrm{~dB}$ if the 417 BER of $10^{-4}$ is considered, even when a higher $\rho_{\text {US }}$ associated with a 418 higher $n_{t}$ is considered.

Fig. 4 portrays the BER achieved by the different signal detectors as 420 a function of the SNR in the context of the proposed LS-SM-MIMO 421 for $K=8, M_{\mathrm{RF}}=18, M=64, n_{t}=4, \rho_{\mathrm{BS}}=0.5$, and $\rho_{\mathrm{US}}=0,422$ where the direct $\mathrm{AE}$ selection scheme is also considered. In Fig. 4, 423 we also characterize the 'oracle-assisted' LS-based signal detector 424 relying on the assumption that the spatial constellation symbol is 425 perfectly known at the BS for the proposed LS-SM-MIMO scheme 426 associated with $J=2,64-\mathrm{QAM}$ as well as for the MMSE-based 427 LS-MIMO detector in conjunction with 64-QAM, where both of them 428 only consider the BER of the classic signal constellation symbol. Here, 429 we assume that the LS-MIMO arrangement uses the same number 430 of RF chains to serve eight single-antenna users communicating 431 over uncorrelated Rayleigh-fading channels. The superiority of our 432 SCS-based MUD over the MMSE- and CS-based signal detectors 433 becomes clear.

Moreover, the performance gap between the oracle LS-based signal 435 detector associated with $7 \mathrm{bpcu}$ and the proposed SCS-based MUD 436 with 7 bpcu is less than $0.5 \mathrm{~dB}$. Note again that the oracle LS-based sig- 437 nal detector only considers the BER of the classic signal constellation 438 


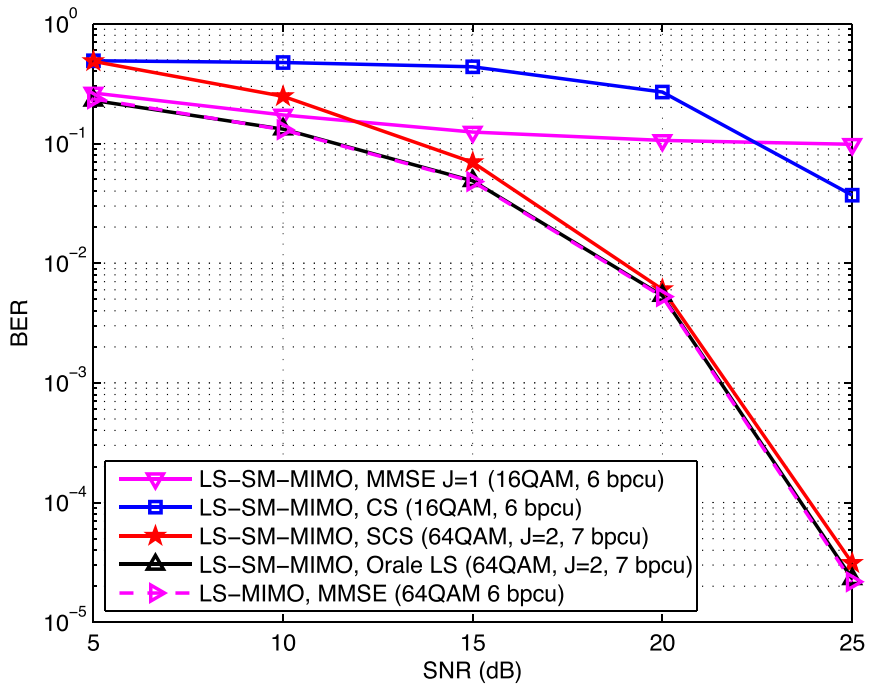

Fig. 4. Total BERs achieved by different signal detectors against different SNRs in the proposed LS-SM-MIMO and conventional LS-MIMO.

439 symbol, whereas the proposed SCS-based MUD considers both the 440 spatial and the classic signal constellation symbols. Finally, compared 441 with the conventional LS-MIMO using the MMSE-based signal de442 tector (6 bpcu), our proposed UL LS-SM-MIMO and the associated 443 SCS-based MUD (7 bpcu) only suffer from a negligible BER loss, 444 which explicitly confirmed the improved UL throughput of the pro445 posed LS-SM-MIMO scheme.

446

\section{CONCLUSION}

447 We have proposed an LS-SM-MIMO scheme for the UL transmis448 sion. The BS employs a large number of AEs but a much smaller 449 number of RF chains, where a simple receive AE selection scheme is 450 used for the improved performance. Each user equipped with multiple 451 AEs but only a single RF chain uses CPSC to combat multipath chan452 nels. SM has been adopted for the UL transmission to improve the UL 453 throughput. The proposed scheme is particularly suitable for scenarios 454 where a large number of low-cost AEs can be accommodated, and 455 both power consumption and hardware cost are heavily determined 456 by the number of RF chains. Due to the reduced number of RF chains 457 at the BS and multiple AEs employed by each user, the UL multiuser 458 signal detection is a challenging large-scale underdetermined problem. 459 We have proposed a joint SM transmission scheme at the users to 460 introduce the group sparsity of multiple aggregate SM signals, and a matching SCS-based MUD at the BS has been proposed to leverage the 461 inherently distributed sparsity of the aggregate SM signal as well as the 462 group sparsity of multiple aggregate SM signals for reliable multiuser 463 signal detection performance. The proposed SCS-based MUD enjoys 464 the low complexity, and our simulation results have demonstrated that 465 it performs better than its conventional counterparts with even much 466 higher UL throughput.

\section{REFERENCES}

468

[1] M. D. Renzo, H. Haas, A. Ghrayeb, S. Sugiura, and L. Hanzo, "Spatial 469 modulation for generalized MIMO: Challenges, opportunities and 470 implementation," Proc. IEEE, vol. 102, no. 1, pp. 56-103, Jan. 2014.

[2] A. Younis, R. Mesleh, M. Di Renzo, and H. Haas, "Generalised spatial 472 modulation for large-scale MIMO," in Proc. EUSIPCO, Sep. 2014, 473 pp. 346-350.

[3] S. Ganesan, R. Mesleh, H. Haas, C. Ahn, and S. Yun, "On the performance 475 of spatial modulation OFDM," in Proc. 40th Asilomar Conf. Signals, Syst. 476 Comput., Oct. 2006, pp. 1825-1829.

[4] F. Rusek et al. "Scaling up MIMO: Opportunities and challenges with 478 very large arrays," IEEE Signal Process. Mag., vol. 30, no. 1, pp. 40-60, 479 Jan. 2013.

[5] N. Serafimovski et al., "Practical implementation of spatial modulation," 481 IEEE Trans. Veh. Technol., vol. 62, no. 9, pp. 4511-4523, Nov. 2013.482

[6] P. Som and A. Chockalingam, "Spatial modulation and space shift 483 keying in single carrier" in Proc. IEEE Int. Symp. PIMRC, Sep. 2012, 484 pp. 1062-1067.

[7] X. Wu, M. Di Renzo, and H. Haas, "Adaptive selection of antennas for 486 optimum transmission in spatial modulation," IEEE Trans. Wireless 487 Commun., vol. 14, no. 7, pp. 3630-3641, Jul. 2015. 488

[8] S. Narayanan et al., "Multi-user spatial modulation MIMO" in Proc. IEEE 489 WCNC, Apr. 2014, pp. 671-676.

[9] X. Wu, H. Claussen, M. D. Renzo, and H. Haas, "Channel estimation 491 for spatial modulation," IEEE Trans. Commun., vol. 62, no. 12, 492 pp. 4362-4372, Dec. 2014

[10] A. Younis, S. Sinanovic, M. Di Renzo, R. Mesleh, and H. Haas, 494 "Generalised sphere decoding for spatial modulation," IEEE Trans. 495 Commun., vol. 61, no. 7, pp. 2805-2815, Jul. 2013.

[11] W. Liu, N. Wang, M. Jin, and H. Xu, "Denoising detection for the gener- 497 alized spatial modulation system using sparse property," IEEE Commun. 498 Lett., vol. 18, no. 1, pp. 22-25, Jan. 2014.

[12] B. Shim, S. Kwon, and B. Song "Sparse detection with integer constraint 500 using multipath matching pursuit," IEEE Commun. Lett., vol. 18, no. 10, 501 pp. 1851-1854, Oct. 2014.

[13] C. Yu et al., "Compressed sensing detector design for space shift keying 503 in MIMO systems," IEEE Commun. Lett., vol. 16, no. 10, pp. 1556-1559, 504 Oct. 2012.

[14] M. F. Duarte and Y. C. Eldar, "Structured compressed sensing: From 506 theory to applications," IEEE Trans. Signal Process., vol. 59, no. 9, 507 pp. 4053-4085, Sep. 2011.

[15] W. Dai and O. Milenkovic, "Subspace pursuit for compressive sens- 509 ing signal reconstruction," IEEE Trans. Inf. Theory, vol. 55, no. 5, 510 pp. 2230-2249, May 2009.

[16] A. Björck, Numerical Methods for Matrix Computations. Cham, 512 Switzerland: Springer Int. Publ. AG, 2014. 
AUTHOR QUERY

NO QUERY. 


\section{Correspondence}

\section{Compressive-Sensing-Based Multiuser Detector for the 2 Large-Scale SM-MIMO Uplink}

3 Zhen Gao, Linglong Dai, Zhaocheng Wang, Senior Member, IEEE, 4 Sheng Chen, Fellow, IEEE, and Lajos Hanzo, Fellow, IEEE

5 Abstract-Conventional spatial modulation (SM) is typically consid6 ered for transmission in the downlink of small-scale multiple-input7 multiple-output (MIMO) systems, where a single antenna element (AE) of 8 a set of, e.g., $2^{p}$ AEs is activated for implicitly conveying $p$ bits. By contrast, 9 inspired by the compelling benefits of large-scale MIMO (LS-MIMO) 10 systems, here, we propose an LS-SM-MIMO scheme for the uplink (UL), 11 where each user having multiple AEs but only a single radio frequency 12 (RF) chain invokes SM for increasing the UL throughput. At the same time, 13 by relying on hundreds of AEs and a small number of RF chains, the base 14 station (BS) can simultaneously serve multiple users while reducing the 15 power consumption. Due to the large number of AEs of the UL users and 16 the comparably small number of RF chains at the BS, the UL multiuser sig17 nal detection becomes a challenging large-scale underdetermined problem. 18 To solve this problem, we propose a joint SM transmission scheme and 19 a carefully designed structured compressive sensing (SCS)-based multi20 user detector (MUD) to be used at the users and the BS, respectively. 21 Additionally, the cyclic-prefix single carrier (CPSC) is used to combat 22 the multipath channels, and a simple receive $\mathrm{AE}$ selection is used for the 23 improved performance over correlated Rayleigh-fading MIMO channels. 24 We demonstrate that the aggregate SM signal consisting of multiple UL 25 users' SM signals of a CPSC block exhibits distributed sparsity. Moreover, 26 due to the joint SM transmission scheme, aggregate SM signals in the same 27 transmission group exhibit group sparsity. By exploiting these intrinsically 28 sparse features, the proposed SCS-based MUD can reliably detect the 29 resultant SM signals with low complexity. Simulation results demonstrate 30 that the proposed SCS-based MUD achieves a better signal detection 31 performance than its counterparts even with higher UL throughput.

32 Index Terms-Compressive sensing (CS), large-scale multiple-input33 multiple-output (LS-MIMO), multiuser detector (MUD), spatial modula34 tion (SM).

\section{INTRODUCTION}

36 A widely recognized consensus is that fifth-generation (5G) sys37 tems will be capable of providing significant energy efficiency and 38 system capacity improvements [1], [2]. Promising techniques, such 39 as large-scale multiple-input-multiple-output (LS-MIMO) and spatial 40 modulation (SM)-MIMO systems, are considered as potent candidates 41 for 5G systems [1]-[5]. LS-MIMO employing hundreds of antenna

Manuscript received May 7, 2015; revised October 1, 2015; accepted November 14, 2015. This work was supported in part by the International Science and Technology Cooperation Program of China under Grant 2015DFG12760, by the National Natural Science Foundation of China under Grant 61571270 and Grant 61201185, by the Beijing Natural Science Foundation under Grant 4142027, and by the Foundation of Shenzhen government. The review of this paper was coordinated by Dr. Y. Xin.

Z. Gao, L. Dai, and Z. Wang are with the Tsinghua National Laboratory for Information Science and Technology (TNList), Department of Electronic Engineering, Tsinghua University, Beijing 100084, China (e-mail: gao-z11@mails. tsinghua.edu.cn; daill@mail.tsinghua.edu.cn; zcwang@mail.tsinghua.edu.cn).

S. Chen and L. Hanzo are with the School of Electronics and Computer Science, University of Southampton, Southampton SO17 1BJ, U.K. (e-mail: sqc@ecs.soton.ac.uk; 1h@ecs.soton.ac.uk).

Color versions of one or more of the figures in this paper are available online at http://ieeexplore.ieee.org.

Digital Object Identifier 10.1109/TVT.2015.2501460 elements (AEs) at the base station (BS) is capable of improving 42 spectral efficiency by orders of magnitude, but it suffers from the 43 nonnegligible power consumption and hardware cost due to one spe- 44 cific radio frequency ( $\mathrm{RF}$ ) chain usually required by every $\mathrm{AE}$ [5]. 45 By using a reduced number of RF chains, the emerging SM-MIMO 46 activates part of available AEs to transmit extra information in the 47 spatial domain, and it has attracted much attention due to its high en- 48 ergy efficiency and reduced hardware cost [5]. However, conventional 49 SM-MIMO is usually considered in the downlink of small-scale 50 MIMO systems, and therefore, its achievable capacity is limited. Indi- 51 vidually, both technologies have their own advantages and drawbacks. 52 By an effective combination of them, one can envision the win-win 53 situation. SM-MIMO is attractive for LS-MIMO systems, since the 54 reduced number of required RF chains in SM-MIMO can reduce the 55 power consumption and hardware cost in conventional LS-MIMO 56 systems. Moveover, hundreds of AEs used in LS-MIMO can im- 57 prove the system throughput of SM-MIMO. Such reciprocity enables 58 LS-MIMO and SM-MIMO to enjoy the apparent compatibility.

In this paper, we propose an LS-SM-MIMO scheme for intrinsi- 60 cally amalgamating the compelling benefits of both LS-MIMO and 61 SM-MIMO for the 5G uplink (UL) over frequency-selective fading 62 channels. In the proposed scheme, each UL user equipped with mul- 63 tiple AEs but only a single RF chain invokes SM for increasing the 64 UL throughput, and the cyclic-prefix single-carrier (CPSC) transmis- 65 sion scheme is adopted to combat the multipath channels [6]. At the 66 BS, hundreds of AEs but only dozens of RF chains are employed to 67 simultaneously serve multiple users, and a direct AE selection scheme 68 is used to improve the system performance over correlated Rayleigh- 69 fading MIMO channels at the BS [7]. The proposed scheme can 70 be adopted in conventional LS-MIMO as a specific UL-transmission 71 mode for reducing the power consumption or, alternatively, for energy- 72 and cost-efficient LS-SM-MIMO, where joint benefits of efficient AE 73 selection [7], transmit precoding [8], and channel estimation [9] can 74 be readily exploited. To sum up, the proposed scheme inherits the 75 advantages of LS-MIMO and SM-MIMO, while reducing the power 76 consumption and hardware cost.

A challenging problem in the proposed UL LS-SM-MIMO scheme 78 is how to realize a reliable multiuser detector (MUD) with low com- 79 plexity. The optimal maximum likelihood (ML) signal detector suffers 80 from excessive complexity. Conventional sphere decoding detectors 81 cannot be readily used in multiuser scenarios and may still exhibit high 82 complexity for LS-SM-MIMO [10]. Existing low-complexity linear 83 signal detectors, e.g., the minimum mean square error (MMSE)-based 84 signal detector, perform well for conventional LS-MIMO systems 85 [4]. However, they are unsuitable for the proposed LS-SM-MIMO 86 UL transmission, since the large number of transmit AEs of the UL 87 users and the reduced number of receive RF chains at the BS make 88 UL multiuser signal detection a large-scale underdetermined/rank- 89 deficient problem. The authors in [11]-[13] proposed compressive 90 sensing (CS)-based signal detectors to solve the underdetermined 91 signal detection problem in SM-MIMO systems, but they only consid- 92 ered the single-user small-scale SM-MIMO systems in the downlink. 93

Against this background, our new contribution is that we exploit 94 the specific signal structure in the proposed multiuser LS-SM-MIMO 95 UL transmission, where each user only activates a single AE in each 96 time slot. Hence, the SM signal of each UL user is sparse with 97 
98 the sparsity level of one, and the aggregate SM signal consisting 99 of multiple UL users' SM signals of a CPSC block exhibits certain 100 distributed sparsity, which can be beneficially exploited for improving 101 the signal detection performance at the BS. Moreover, we propose 102 a joint SM transmission scheme for the UL users in conjunction 103 with an appropriately structured CS (SCS)-based MUD at the BS. 104 The proposed SCS-based MUD is specifically tailored to leverage 105 the inherently distributed sparsity of the aggregate SM signal and the 106 group sparsity of multiple aggregate SM signals, owing to the joint SM 107 transmission scheme for reliable signal detection performance. Our 108 simulation results demonstrate that the proposed SCS-based MUD is 109 capable of outperforming the conventional detectors even with higher 110 UL throughput.

111 The rest of this paper is organized as follows. Section II introduces 112 the system model of the proposed LS-SM-MIMO scheme. Section III 113 specifies the proposed joint SM transmission and SCS-based MUD. 114 Section IV provides our simulation results. Section V concludes this 115 paper.

116 Throughout this paper, lowercase and uppercase boldface letters 117 denote vectors and matrices, respectively, whereas $(\cdot)^{T},(\cdot)^{*},(\cdot)^{\dagger}$, and $118\lfloor\cdot\rfloor$ denote the transpose, conjugate transpose, Moore-Penrose matrix 119 inversion, and the integer floor operators, respectively. The $l_{0}$ and $120 l_{2}$ norm operations are given by $\|\cdot\|_{0}$ and $\|\cdot\|_{2}$, respectively. The 121 support set of vector $\mathbf{x}$ is denoted by $\operatorname{supp}\{\mathbf{x}\}$, and $\mathbf{x}\rangle_{i}$ denotes the $i$ th 122 entry of vector $\mathbf{x}$. Additionally, $\left.\mathbf{x}\right|_{\Gamma}$ denotes the entries of $\mathbf{x}$ defined 123 in the set $\Gamma,\left.\boldsymbol{\Phi}\right|_{\Gamma}$ denotes the submatrix whose columns comprise the 124 columns of $\boldsymbol{\Phi}$ that are defined in $\Gamma$, and $\boldsymbol{\Phi}\rangle_{\Gamma}$ denotes the submatrix 125 whose rows comprise the rows of $\boldsymbol{\Phi}$ that are defined in $\Gamma$. The 126 expectation operator is given by $E\{\cdot\} . \bmod (x, y)=x-\lfloor x / y\rfloor y$ if $127 y \neq 0$ and $x-\lfloor x / y\rfloor y \neq 0$, whereas $\bmod (x, y)=y$ if $y \neq 0$ and $128 x-\lfloor x / y\rfloor y=0$.

\section{SySTEM MODEL}

130 We first introduce the proposed LS-SM-MIMO scheme and then 131 focus our attention on the UL transmission with an emphasis on the 132 multiuser signal detection.

\section{A. Proposed Multiuser LS-SM-MIMO Scheme}

134 As shown in Fig. 1, we consider the proposed LS-SM-MIMO from 135 both the BS side and the user side. For conventional LS-MIMO, the 136 number of AEs employed by the BS is equal to the number of its RF 137 chains [4]. However, the BS in LS-SM-MIMO, as shown in Fig. 1, 138 is equipped with a much smaller number of RF chains $M_{\mathrm{RF}}$ than 139 the total number of AEs $M$, i.e., we have $M_{\mathrm{RF}} \ll M$. Conventional 140 LS-MIMO systems typically assume single-antenna users [4]. By 141 contrast, in the proposed scheme, each user is equipped with $n_{t}>1$ 142 AEs but only a single RF chain, and SM is adopted for the UL 143 transmission, where only one of the available AEs is activated for 144 data transmission. It has been shown that the main power consumption 145 and hardware cost of cellular networks comes from the radio access 146 network [1]. Hence, using a reduced number of expensive RF chains 147 compared with the total number of AEs at the BS can substantially 148 reduce both the power consumption and the hardware cost for the 149 operators. Meanwhile, it is feasible to incorporate several AEs and a 150 single RF chain in the handsets. The resultant increased degrees of 151 freedom in the spatial domain may then be exploited for improving 152 the UL throughput. The proposed scheme can be considered as an 153 optional UL-transmission mode in conventional LS-MIMO systems, 154 where AE selection schemes may be adopted for beneficially selecting 155 the most suitable $M_{\mathrm{RF}}$ AEs at the BS to receive UL SM signals [7]. 156 Alternatively, it can also be used for the UL of LS-SM-MIMO, when

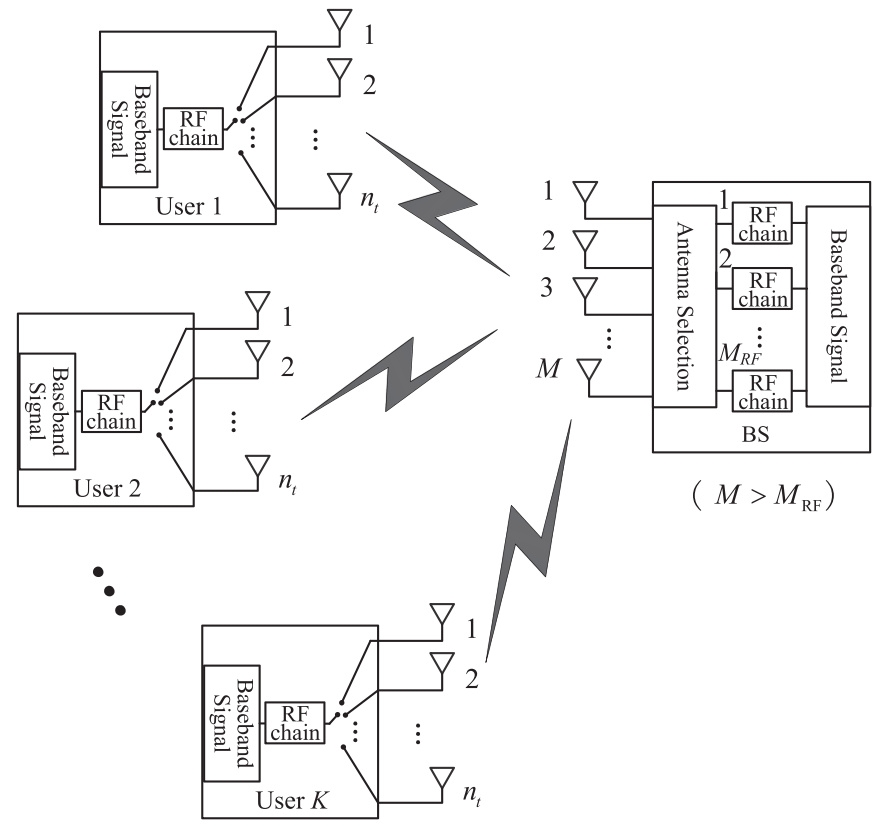

Fig. 1. In the proposed UL LS-SM-MIMO, the BS is equipped with $M$ AEs and $M_{\mathrm{RF}} \mathrm{RF}$ chains to simultaneously serve $K$ users, where $M \gg M_{\mathrm{RF}}>$ $K$, and each user is equipped with $n_{t}>1$ AEs and one RF chain. By exploiting the improved degree of freedom in the spatial domain, multiple users can simultaneously exploit SM for improving the UL throughput.

advantageously combining transmit precoding, receive AE selection, 157 and channel estimation [7]-[9].

\section{B. Uplink Multiuser Transmission}

We first consider the generation of SM signals at the users. The SM 160 signal $\mathbf{x}_{k}=\mathbf{e}_{k} s_{k}$ transmitted by the $k$ th user in a time slot consists 161 of two parts: the spatial constellation symbol $\mathbf{e}_{k} \in \mathbb{C}^{n_{t}}$ and the signal 162 constellation symbol $s_{k} \in \mathbb{C}$. $\mathbf{e}_{k}$ is generated by mapping $\left\lfloor\log _{2}\left(n_{t}\right)\right\rfloor 163$ bits to the index of the active $\mathrm{AE}$, and typically, the user terminal 164 employs $n_{t}=2^{p}$ AEs, where $p$ is a positive integer. Due to only a 165 single RF chain employed at each user, only one entry of $\mathbf{e}_{k}$ associated 166 with the active $\mathrm{AE}$ is equal to one, and the rest of the entries of $\mathbf{e}_{k}$ are 167 zeros, i.e., we have

$$
\operatorname{supp}\left(\mathbf{e}_{k}\right) \in \mathbb{A}, \quad\left\|\mathbf{e}_{k}\right\|_{0}=1, \quad\left\|\mathbf{e}_{k}\right\|_{2}=1
$$

where $\mathbb{A}=\left\{1,2, \ldots, n_{t}\right\}$ is the spatial constellation symbol set. The 169 signal constellation symbol comes from $L$-ary modulation, i.e., $s_{k} \in 170$ $\mathbb{L}$, where $\mathbb{L}$ is the signal constellation symbol set [e.g., 64 quadrature 171 amplitude modulation (QAM)] of size $L$. Hence, each UL user's SM 172 signal carries the information of $\log _{2}(L)+\log _{2}\left(n_{t}\right)$ bits per channel 173 use (bpcu), and the overall UL throughput is $K\left(\log _{2}(L)+\log _{2}\left(n_{t}\right)\right) 174$ bpcu. The users rely on the CPSC scheme for transmitting their SM 175 signals [6]. Explicitly, each CPSC block consists of a cyclic prefix 176 (CP) having the length of $P-1$ and the associated data block having 177 the length of $Q$. Hence, the length of each CPSC block is $Q+P-1,178$ where this $\mathrm{CP}$ is capable of counteracting a dispersive multipath 179 channel imposing dispersion over $P$ samples. The concatenated data 180 block consists of $Q$ successive SM signals.

At the receiver, due to the reduced number of RF chains at the BS, 182 only $M_{\mathrm{RF}}$ receive AEs can be exploited to receive signals, where exist- 183 ing receive AE selection schemes can be adopted to preselect $M_{\mathrm{RF}}$ re- 184 ceive AEs for achieving an improved signal detection performance [7]. 185 Since the BS can serve $K$ users simultaneously, after the removal of 186 
187 the $\mathrm{CP}$, the received signal $\mathbf{y}_{q} \in \mathbb{C}^{M_{\mathrm{RF}}}$ for $1 \leq q \leq Q$ of the $q$ th time 188 slot of a specific CPSC block can be expressed as

$$
\begin{aligned}
\mathbf{y}_{q}=\sum_{k=1}^{K} \mathbf{y}_{k, q}+ & \left.\mathbf{w}_{q}=\sum_{p=0}^{P-1} \sum_{k=1}^{K} \mathbf{H}_{k, p}\right\rangle_{\Theta} \mathbf{x}_{k,} \bmod (q-p, Q) \\
+\mathbf{w}_{q} & =\sum_{p=0}^{P-1} \sum_{k=1}^{K} \tilde{\mathbf{H}}_{k, p} \mathbf{x}_{k,} \bmod (q-p, Q)+\mathbf{w}_{q}
\end{aligned}
$$

189 where $\mathbf{H}_{k, p} \in \mathbb{C}^{M \times n_{t}}$ is the $k$ th user's MIMO channel matrix for the $190 p$ th multipath component, i.e., $\left.\mathbf{H}_{k, p}\right\rangle_{\Theta}=\tilde{\boldsymbol{H}}_{k, p} \in \mathbb{C}^{M_{\mathrm{RF}} \times n_{t}}$; the set $191 \Theta$ is determined by the AE selection scheme used; the elements of $192 \Theta$ having the cardinality of $M_{\mathrm{RF}}$ are uniquely selected from the set $193\{1,2, \ldots, M\} ; \mathbf{x}_{k, q}$ has one nonzero entry; and $\mathbf{w}_{q} \in \mathbb{C}^{M_{\mathrm{RF}}}$ is the 194 additive white Gaussian noise (AWGN) vector with entries obeying 195 the independent and identically distributed (i.i.d.) circularly symmet196 ric complex Gaussian distribution with zero mean and a variance 197 of $\sigma_{w}^{2} / 2$ per dimension, which is denoted by $\mathcal{C N}\left(0, \sigma_{w}^{2}\right) . \mathbf{H}_{k, p}=$ $198 \mathbf{R}_{\mathrm{BS}}^{1 / 2} \overline{\boldsymbol{H}}_{k, p} \mathbf{R}_{\mathrm{US}}^{1 / 2}$, the entries of $\overline{\boldsymbol{H}}_{k, p}$ obey the i.i.d. $\mathcal{C N}(0,1)$, and $199 \mathbf{R}_{\mathrm{US}}$ with the correlation coefficient $\rho_{\mathrm{US}}$ and $\mathbf{R}_{\mathrm{BS}}$ with the correlation 200 coefficient $\rho_{\mathrm{BS}}$ are correlation matrices at the users and the BS, 201 respectively. The specific element of the $m$ th row and the $n$th column 202 of $R_{\mathrm{BS}}\left(R_{\mathrm{US}}\right)$ is $\rho_{\mathrm{BS}}^{|m-n|}\left(\rho_{\mathrm{US}}^{|m-n|}\right)$. For correlated Rayleigh-fading 203 MIMO channels, the specific $\Theta$ or receive AE selection scheme has 204 an impact on the attainable system performance. In this paper, the 205 direct $\mathrm{AE}$ selection scheme is used for maximizing the minimum 206 geometric distance between any pair of the selected AEs [7]. For 207 uniform linear arrays (ULAs), $\Theta=\left\{\varphi+m_{\mathrm{RF}}\left\lfloor M / M_{\mathrm{RF}}\right\rfloor\right\}_{m_{\mathrm{RF}}=0}^{M_{\mathrm{RF}}-1}$ 208 with $1 \leq \varphi \leq\left\lfloor M / M_{\mathrm{RF}}\right\rfloor-1$. Then, (2) can be further expressed as

$$
\mathbf{y}_{q}=\sum_{p=0}^{P-1} \tilde{\mathbf{H}}_{p} \mathbf{x} \bmod (q-p, Q)+\mathbf{w}_{q}
$$

209 by defining $\tilde{\boldsymbol{H}}_{p}=\left[\tilde{\boldsymbol{H}}_{1, p} \tilde{\boldsymbol{H}}_{2, p}, \ldots, \tilde{\boldsymbol{H}}_{K, p}\right] \in \mathbb{C}^{M_{\mathrm{RF}} \times\left(n_{t} K\right)}$ and $210 \mathbf{x}_{q}=\left[\left(\mathbf{x}_{1, q}\right)^{T}\left(\mathbf{x}_{2, q}\right)^{T}, \ldots,\left(\mathbf{x}_{K, q}\right)^{T}\right]^{T} \in \mathbb{C}^{\left(n_{t} K\right)}$. By considering 211 the $Q$ SM signals of a specific CPSC block, we arrive at

$$
\mathbf{y}=\tilde{\mathbf{H}} \mathbf{x}+\mathbf{w}
$$

212 where $\mathbf{y}=\left[\left(\mathbf{y}_{1}\right)^{T}\left(\mathbf{y}_{2}\right)^{T}, \ldots,\left(\mathbf{y}_{Q}\right)^{T}\right]^{T} \in\left(_{\mathrm{RF}}^{\left(M_{\mathrm{R}}\right)}\right.$, the aggregate $213 \mathrm{SM}$ signal $\mathbf{x}=\left[\left(\mathbf{x}_{1}\right)^{T}\left(\mathbf{x}_{2}\right)^{T}, \ldots,\left(\mathbf{x}_{Q}\right)^{T}\right]^{T} \in{ }^{\left(K n_{t} Q\right)}, \quad \mathbf{w}=$ $214\left[\left(\mathbf{w}_{1}\right)^{T}\left(\mathbf{w}_{2}\right)^{T}, \ldots,\left(\mathbf{w}_{Q}\right)^{T}\right]^{T}$, and

$\tilde{\mathbf{H}}=\left[\begin{array}{cccccc}\tilde{\mathbf{H}}_{0} & \mathbf{0} & \mathbf{0} & \ldots & \tilde{\mathbf{H}}_{2} & \tilde{\mathbf{H}}_{1} \\ \tilde{\mathbf{H}}_{1} & \tilde{\mathbf{H}}_{0} & \mathbf{0} & \ldots & \vdots & \tilde{\mathbf{H}}_{2} \\ \vdots & \tilde{\mathbf{H}}_{1} & \tilde{\mathbf{H}}_{0} & \ldots & \tilde{\mathbf{H}}_{P-1} & \vdots \\ \tilde{\mathbf{H}}_{P-1} & \vdots & \tilde{\mathbf{H}}_{1} & \ldots & \mathbf{0} & \tilde{\mathbf{H}}_{P-1} \\ \mathbf{0} & \tilde{\mathbf{H}}_{P-1} & \vdots & \vdots & \vdots & \mathbf{0} \\ \vdots & \mathbf{0} & \tilde{\mathbf{H}}_{P-1} & \vdots & \vdots & \vdots \\ \vdots & \vdots & \mathbf{0} & \vdots & \mathbf{0} & \vdots \\ \vdots & \vdots & \vdots & \vdots & \tilde{\mathbf{H}}_{0} & \mathbf{0} \\ \mathbf{0} & \mathbf{0} & \mathbf{0} & \cdots & \tilde{\mathbf{H}}_{1} & \tilde{\mathbf{H}}_{0}\end{array}\right]_{(5)}$

215 The signal-to-noise ratio (SNR) at the receiver is defined by SNR = $216 E\left\{\|\tilde{\boldsymbol{H}} \mathbf{x}\|_{2}^{2}\right\} / E\left\{\|\mathbf{w}\|_{2}^{2}\right\}$.
The optimal signal detector for (4) relies on the ML algorithm, i.e., 217

$$
\begin{aligned}
\min _{\hat{\mathbf{x}}} & \|\mathbf{y}-\tilde{\mathbf{H}} \hat{\mathbf{x}}\|_{2}=\min _{\left\{\hat{\mathbf{x}}_{k, q}\right\}_{k=1, q=1}^{K, Q}}\|\mathbf{y}-\tilde{\mathbf{H}} \hat{\mathbf{x}}\|_{2} \\
\text { s.t. } & \left.\operatorname{supp}\left(\hat{\mathbf{x}}_{k, q}\right) \in \mathbb{A}, \hat{\mathbf{x}}_{k, q}\right\rangle_{\operatorname{supp}\left(\hat{\mathbf{x}}_{k, q}\right)} \in \mathbb{L}, 1 \leq k \leq K, 1 \leq q \leq Q
\end{aligned}
$$

whose complexity exponentially increases with the number of users, 218 since the size of the search set for the ML detector is $\left(n_{t} \cdot L\right)^{K Q} .219$ This excessive complexity can be unaffordable in practice. To reduce 220 the complexity, near-optimal sphere decoding detectors have been 221 proposed [10], but their complexity may still remain high, particularly 222 for the systems supporting large $K, Q, n_{t}$, and $L$ [11]. In conventional 223 LS-MIMO systems, low-complexity linear signal detectors (e.g., the 224 MMSE-based signal detector) have been shown to be near optimal 225 since $M=M_{\mathrm{RF}} \gg K$ and $n_{t}=1$ make multiuser signal detection 226 an overdetermined problem [4]. However, in the proposed scheme, we 227 have $M_{\mathrm{RF}}<K n_{t}$. Hence, the multiuser signal detection problem (6) 228 represents a large-scale underdetermined problem. Consequently, the 229 conventional linear signal detectors perform poorly in the proposed 230 LS-SM-MIMO [11]. By exploiting the sparsity of SM signals, the 231 authors in [11]-[13] have proposed the concept of CS-based signal 232 detectors for the downlink of small-scale SM-MIMO operating in a 233 single-user scenario. However, these signal detectors are unsuitable 234 for the proposed multiuser scenarios. Observe from (1) that $\mathbf{x}_{k, q}$ is 235 a sparse signal having a sparsity level of one. Hence, the aggregate 236 SM signal $\mathbf{x}$, which consists of multiple users' SM signals in $Q 237$ time slots, exhibits distributed sparsity with the sparsity level of $K Q .238$ This property of $\mathbf{x}$ inspires us to exploit the SCS theory for the 239 multiuser signal detection [14]. To further improve the signal detection 240 performance and to increase the system's throughput, we propose a 241 joint SM transmission scheme and an SCS-based MUD, which will be 242 detailed in the following section.

\section{SCS-BASED MUD FOR LS-SM-MIMO UL}

To solve the multiuser signal detection of our UL LS-SM-MIMO 245 system, we first propose a joint SM transmission scheme to be 246 employed at the users. Accordingly, an SCS-based low-complexity 247 MUD is developed at the BS, whereby the distributed sparsity of the 248 aggregate SM signal and the group sparsity of multiple aggregate SM 249 signals are exploited. Moreover, the computational complexity of the 250 proposed SCS-based MUD is discussed.

\section{A. Joint SM Transmission Scheme at the Users}

For the $k$ th user in the $q$ th time slot, every successive $J$ CPSC block 253 is considered as a group, and they share the same spatial constellation 254 symbol, i.e.,

$$
\begin{aligned}
\operatorname{supp}\left(\mathbf{x}_{k, q}^{(1)}\right)=\operatorname{supp}\left(\mathbf{x}_{k, q}^{(2)}\right)=\cdots= & \operatorname{supp}\left(\mathbf{x}_{k, q}^{(J)}\right), \\
& 1 \leq k \leq K, 1 \leq q \leq Q
\end{aligned}
$$

where we introduce the superscript $(j)$ to denote the $j$ th CPSC block, 256 and $J$ is typically small, e.g., $J=2$. In CS theory, the specific signal 257 structure, where $\mathbf{x}_{k, q}^{(1)}, \mathbf{x}_{k, q}^{(2)}, \ldots, \mathbf{x}_{k, q}^{(J)}$ share a common support, is often 258 referred to as the group sparsity. Similarly, the aggregate SM signals 259 consisting of the $K$ users' SM signals also exhibit group sparsity, i.e., 260

$$
\operatorname{supp}\left(\mathbf{x}^{(1)}\right)=\operatorname{supp}\left(\mathbf{x}^{(2)}\right)=\cdots=\operatorname{supp}\left(\mathbf{x}^{(J)}\right) .
$$

Although exhibiting group sparsity may slightly reduce the informa- 261 tion carried by the spatial constellation symbols, it is also capable of 262 
263 reducing the number of the RF chains required according to the SCS 264 theory, while simultaneously improving the total bit error rate (BER) 265 of the entire system even with higher UL throughput. This conclusion 266 will be confirmed by our simulation results.

\section{B. SCS-Based MUD at the BS}

268 According to (4), the received signals at the BS in the same group 269 can be expressed as

$$
\mathbf{y}^{(j)}=\tilde{\mathbf{H}}^{(j)} \mathbf{x}^{(j)}+\mathbf{w}^{(j)}, \quad 1 \leq j \leq J
$$

270 where $\mathbf{y}^{(j)}$ denotes the received signal in the $j$ th CPSC block, whereas $271 \tilde{\boldsymbol{H}}^{(j)}$ and $\mathbf{w}^{(j)}$ are the effective MIMO channel matrix and the AWGN 272 vector, respectively.

273 The intrinsically distributed sparsity of $\mathbf{x}^{(j)}$ and the underdeter274 mined nature of (9) inspire us to solve the signal detection problem 275 based on CS theory, which can efficiently acquire the sparse solutions 276 to underdetermined linear systems. Moreover, the $J$ different aggre277 gate SM signals in (9) can be jointly exploited for improving the signal 278 detection performance due to the group sparsity of $\left\{\mathbf{x}^{(j)}\right\}_{j=1}^{J}$. Thus, 279 by considering both the distributed sparsity and the group sparsity of 280 the aggregate SM signals, the multiuser signal detection at the BS can 281 be formulated as the following optimization problem:

$$
\begin{array}{cl}
\underset{\left\{\widehat{\mathbf{x}}^{(j)}\right\}_{j=1}^{J}}{\min } & \sum_{j=1}^{J}\left\|\mathbf{y}^{(j)}-\tilde{\mathbf{H}}^{(j)} \widehat{\mathbf{x}}^{(j)}\right\|_{2}^{2}=\min _{\left\{\widehat{\mathbf{x}}_{k, q}^{(j)}\right\}_{j=1, k=1, q=1}^{J, K, Q}} \\
& \sum_{j=1}^{J}\left\|\mathbf{y}^{(j)}-\tilde{\mathbf{H}}^{(j)} \widehat{\mathbf{x}}^{(j)}\right\|_{2}^{2} \\
\text { s.t. } & \left\|\widehat{\mathbf{x}}_{k, q}^{(j)}\right\|_{0}=1,1 \leq j \leq J, 1 \leq q \leq Q, 1 \leq k \leq K .
\end{array}
$$

282 Our proposed SCS-based MUD solves the optimization problem (10) 283 with the aid of two steps. In the first step, we estimate the spatial 284 constellation symbols, i.e., the indexes of $K$ users' active AEs in $J$ 285 successive CPSC blocks. In the second step, we infer the legitimate 286 signal constellation symbols of the $K$ users in $J$ CPSC blocks.

287 1. Step 1-Estimation of Spatial Constellation Symbols: We pro288 pose a group subspace pursuit (GSP) algorithm developed from the 289 classical subspace pursuit (SP) algorithm in [15] to acquire the 290 sparse solution to the large-scale underdetermined problem (10), 291 where both the a priori sparse information (i.e., $\left\|\mathbf{x}_{k, q}^{(j)}\right\|_{0}=1$ ) and 292 the group sparsity of $\mathbf{x}^{(1)}, \mathbf{x}^{(2)}, \ldots, \mathbf{x}^{(J)}$ are exploited for improv293 ing the multiuser signal detection performance. The proposed GSP 294 algorithm is described in Algorithm 1, which estimates SM signal $295\left\{\widehat{\mathbf{x}}_{k, q}^{(j)}\right\}_{k=1, j=1, q=1}^{K, J, Q}$. Hence, the estimated spatial constellation symbol 296 is $\left\{\operatorname{supp}\left(\widehat{\mathbf{x}}_{k, q}^{(j)}\right)\right\}_{k=1, j=1, q=1}^{K, J, Q}$.

\section{Algorithm 1 Proposed GSP Algorithm.}

Input: Noisy received signals $\mathbf{y}^{(j)}$ and effective channel matrices $\tilde{H}^{(j)}$ for $1 \leq j \leq J$

Output:Estimated $\widehat{\mathbf{x}}^{(j)}=\left[\left(\widehat{\mathbf{x}}_{1}^{(j)}\right)^{T}\left(\widehat{\mathbf{x}}_{2}^{(j)}\right)^{T}, \ldots,\left(\widehat{\mathbf{x}}_{Q}^{(j)}\right)^{T}\right]^{T}$, where $\widehat{\mathbf{x}}_{q}^{(j)}=\left[\left(\widehat{\mathbf{x}}_{1, q}^{(j)}\right)^{T}\left(\widehat{\mathbf{x}}_{2, q}^{(j)}\right)^{T}, \ldots,\left(\widehat{\mathbf{x}}_{K, q}^{(j)}\right)^{T}\right]^{T}$ for $1 \leq q \leq Q$.

$1: \mathbf{r}^{(j)}=\mathbf{y}^{(j)}$ for $1 \leq j \leq J ;\{$ Initialization $\}$

$2: \Omega^{0}=\emptyset ;\{$ Empty support set $\}$

$303 \quad 3: t=1 ;\{$ Iteration index $\}$
4: repeat

5: $\quad \mathbf{a}_{k, q}^{(j)}=\left(\tilde{\boldsymbol{H}}_{k, q}^{(j)}\right)^{*} \mathbf{r}^{(j)}$ for $1 \leq k \leq K, 1 \leq q \leq Q$, and $1 \leq j \leq J ; 305$ \{Correlation\}

6: $\left.\quad \tau_{k, q}=\arg \max _{\tau_{k, q}} \sum_{j=1}^{J} \| \mathbf{a}_{k, q}^{(j)}\right\rangle_{\tau_{k, q}} t \|_{2}^{2} \quad$ for $\quad 1 \leq k \leq K, 307$ $1 \leq q \leq Q ;\{$ Identify support $\}$ 308

7: $\quad \Gamma=\left\{\tau_{k, q}+(k-1+K(q-1)) n_{t}\right\}_{k=1, q=1}^{K, Q} ; \quad$ \{Preliminary 309 support set $\}$

8: $\left.\quad \mathbf{b}^{(j)}\right|_{\Omega^{t-1} \cup \Gamma}=\left(\left.\tilde{\boldsymbol{H}}^{(j)}\right|_{\Omega^{t-1} \cup \Gamma}\right)^{\dagger} \mathbf{y}^{(j)}$ for $1 \leq j \leq J ;$ Least 311 squares $\}$

9: $\left.\quad \omega_{k, q}=\arg \max _{\tilde{\omega}_{k, q}} \sum_{j=1}^{J} \| \mathbf{b}_{k, q}^{(j)}\right\rangle_{\widetilde{\omega}_{k, q}} \|_{2}^{2} \quad$ for $\quad 1 \leq k \leq K, 313$ $1 \leq q \leq Q ;\{$ Pruning support set\}

314

10: $\Omega^{t}=\left\{\omega_{k, q}+(k-1+K(q-1)) n_{t}\right\}_{k=1, q=1}^{K, Q} ; \quad$ \{Final 315 support set $\}$

11: $\left.\quad \mathbf{c}^{(j)}\right|_{\Omega^{t}}=\left(\left.\tilde{\boldsymbol{H}}^{(j)}\right|_{\Omega^{t}}\right)^{\dagger} \mathbf{y}^{(j)}$ for $1 \leq j \leq J ;\{$ Least squares $\} \quad 317$

12: $\quad \mathbf{r}^{(j)}=\mathbf{y}^{(j)}-\tilde{\boldsymbol{H}}^{(j)} \mathbf{c}^{(j)}$ for $1 \leq j \leq J ;\{$ Compute residual $\} 318$

13: $t=t+1 ;\{$ Update iteration index $\} \quad 319$

14: until $\Omega^{t}=\Omega^{t-1}$ or $t \geq Q$

Compared with the classical SP algorithm, the proposed GSP algo- 321 rithm exploits the distributed sparsity and group sparsity of $\left\{\mathbf{x}^{(j)}\right\}_{j=1}^{J} .322$ More explicitly, $\mathbf{x}^{(j)} \in \mathbb{C}^{\left(K Q n_{t}\right)}$ consists of the $K Q$ low-dimensional 323 sparse vectors $\mathbf{x}_{k, q}^{(j)} \in \mathbb{C}^{n_{t}}$, where each $\mathbf{x}_{k, q}^{(j)}$ has the known sparsity 324 level of one, and the aggregate SM signals $\mathbf{x}^{(1)}, \mathbf{x}^{(2)}, \ldots, \mathbf{x}^{(J)}$ exhibit 325 group sparsity. Specifically, the differences between the proposed GSP 326 algorithm and the classical SP algorithm lie in the following two 327 aspects: 1) the identification of the support set including the steps 328 of the preliminary support set and the final support set as shown in 329 Algorithm 1; and 2) the joint processing of $\mathbf{y}^{(1)}, \mathbf{y}^{(2)}, \ldots, \mathbf{y}^{(J)}$. First, 330 for the support selection, taking the step of the preliminary support set 331 for instance, when selecting the preliminary support set, the classical 332 SP algorithm selects the support set associated with the first $K Q 333$ largest values of the global correlation result $\left(\tilde{\boldsymbol{H}}^{(j)}\right)^{*} \mathbf{r}^{(j)}$. By contrast, 334 the proposed GSP algorithm selects the support set associated with 335 the largest value from the local correlation result in each $\left(\tilde{\boldsymbol{H}}_{k, q}^{(j)}\right)^{*} \mathbf{r}^{(j)} .336$ This way, the distributed sparsity of the aggregate SM signal can be ex- 337 ploited for improved signal detection performance. Second, compared 338 with the classical SP algorithm, the proposed GSP algorithm jointly 339 exploits the $J$ correlated signals having the group sparsity, which can 340 bring the further improved signal detection performance.

It should be noted that even for the special case of $J=1$, i.e., 342 without using the joint SM transmission scheme, the proposed GSP 343 algorithm still achieves a better signal detection performance than 344 the classical SP algorithm when handling the aggregate SM signal, 345 since the inherently distributed sparsity of the aggregate SM signal is 346 leveraged to improve the signal detection performance.

347

2. Step 2-Acquisition of Signal Constellation Symbols: Following 348 Step 1, we can also acquire a rough estimate of the signal constellation 349 symbol for each user in each time slot. By searching for the minimum 350 Euclidean distance between this rough estimate of the signal constel- 351 lation symbol and the legitimate constellation symbols of $\mathbb{L}$, we can 352 obtain the final estimate of signal constellation symbols.

\section{Computational Complexity}

The optimal ML signal detector has a prohibitively high com- 355 putational complexity of $\mathcal{O}\left(\left(L \cdot n_{t}\right)^{(K \cdot Q)}\right)$ according to (6). The 356 sphere decoding detectors [10] are indeed capable of reducing the 357 computational complexity, but they may still suffer from unaffordable 358 complexity, particularly for large $K, Q, L$, and $n_{t}$ values. By contrast, 359 the conventional MMSE-based detector for LS-MIMO and CS-based 360 


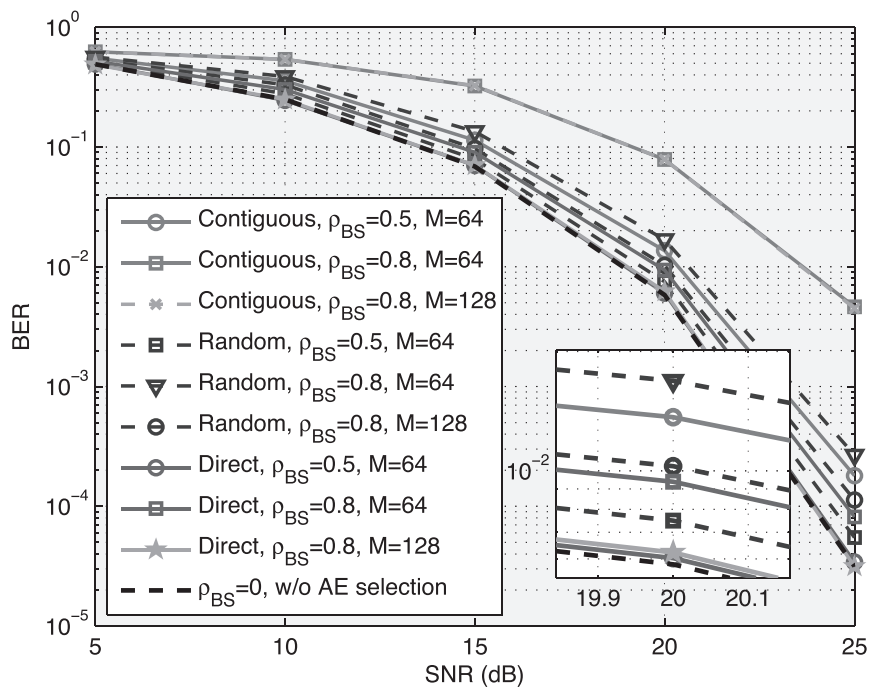

Fig. 2. Total BERs achieved by the proposed SCS-based MUD with different AE selection schemes, where $K=8, J=2$, 64-QAM, $M_{\mathrm{RF}}=18, n_{t}=4$, and $\rho_{\mathrm{US}}=0$ are considered.

361 detector [13] for small-scale SM-MIMO enjoy the low complexity of $362 \mathcal{O}\left(M_{\mathrm{RF}} \cdot\left(n_{t} \cdot Q \cdot K\right)^{2}+\left(n_{t} \cdot Q \cdot K\right)^{3}\right)$ and $\mathcal{O}\left(2 M_{\mathrm{RF}} \cdot(Q \cdot K)^{2}+\right.$ $\left.363(Q \cdot K)^{3}\right)$, respectively. For the proposed SCS-based MUD, most 364 of the computational requirements are imposed by the least squares 365 (LS) operations, which has complexity of $\mathcal{O}\left(J \cdot\left(2 M_{\mathrm{RF}} \cdot(Q \cdot K)^{2}+\right.\right.$ $\left.366(Q \cdot K)^{3}\right)$ ) [16]. Consequently, the computational complexity per 367 CPSC block is $\mathcal{O}\left(2 M_{\mathrm{RF}} \cdot(Q \cdot K)^{2}+(Q \cdot K)^{3}\right)$, since $J$ successive 368 aggregate SM signals are jointly processed. Compared with conven369 tional signal detectors, the proposed SCS-based MUD benefits from 370 substantially lower complexity, and it has similar low complexity as 371 the conventional MMSE- and CS-based signal detectors.

\section{Simulation Results}

373 A simulation study was carried out to compare the attainable perfor374 mance of the proposed SCS-based MUD to that of the MMSE-based 375 signal detector [4] and to that of the CS-based signal detector [13]. 376 In the LS-SM-MIMO system considered, the BS used a ULA relying 377 on a large number of AEs $M$, but a much smaller number of RF 378 chains $M_{\mathrm{RF}}$, whereas $K$ users employing $n_{t}$ AEs but only a single RF 379 chain simultaneously use the CPSC scheme associated with $P=8$ and $380 Q=64$ to transmit the SM signals to the BS. The total BER including 381 both the spatial constellation symbols and the signal constellation 382 symbols was evaluated.

383 Fig. 2 compares the total BERs achieved by the proposed SCS-based 384 MUD with different AE selection schemes, where $K=8, J=2$, 385 64-QAM, $M_{\mathrm{RF}}=18, n_{t}=4$, and $\rho_{\mathrm{US}}=0$ are considered. The con386 tiguous AE selection scheme implies that we select $M_{\mathrm{RF}}$ adjacent 387 AEs, i.e., $\Theta=\left\{\varphi+m_{\mathrm{RF}}\right\}_{m_{\mathrm{RF}}=0}^{M_{\mathrm{RF}}-1}$ with $1 \leq \varphi \leq M-M_{\mathrm{RF}}+1$. By 388 contrast, in the random $\mathrm{AE}$ selection scheme, the elements of $\Theta$ are 389 randomly selected from the set $\{1,2, \ldots, M\}$, whereas the direct 390 AE selection scheme in [7] has been described in Section II-B. 391 Furthermore, the BER achieved by the SCS-based MUD relying on $392 \rho_{\mathrm{BS}}=0$ is also considered as a performance bound, since the choice 393 of $\rho_{\mathrm{BS}}=0$ and $\rho_{\mathrm{US}}=0$ implies the uncorrelated Rayleigh-fading 394 MIMO channels. Observe from Fig. 2 that the direct AE selection 395 scheme outperforms the other pair of AE selection schemes. Moreover, 396 for a certain AE selection scheme, the BER performance degrades 397 when $M_{\mathrm{RF}} / M$ or $\rho_{\mathrm{BS}}$ increases. For the direct AE selection scheme, 398 the BER performance of $\rho_{\mathrm{BS}}=0.8, M=128$ and of $\rho_{\mathrm{BS}}=0.5$,

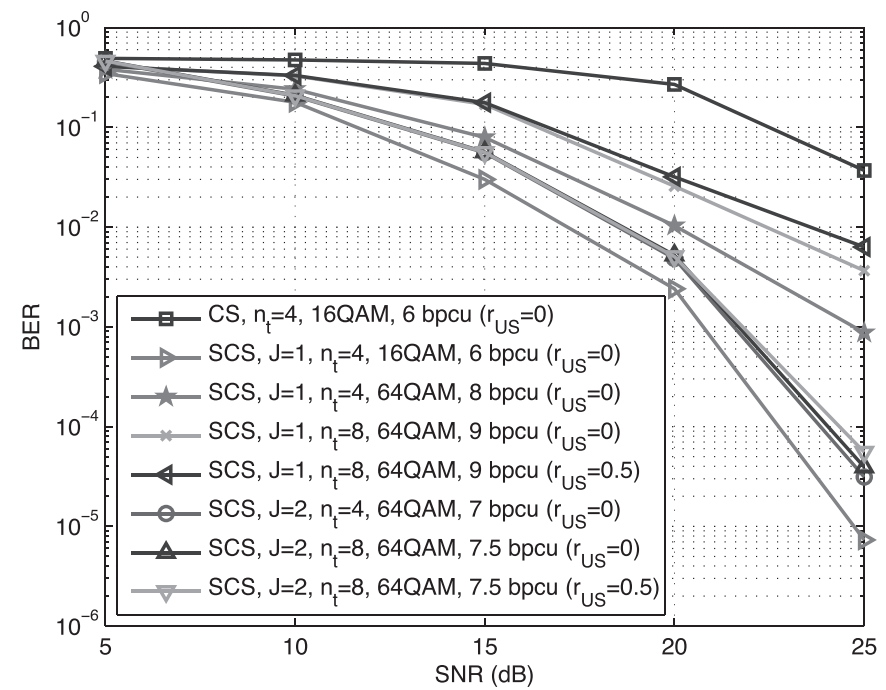

Fig. 3. Total BERs achieved by the CS-based signal detector and the SCS-based MUD against different SNRs in LS-SM-MIMO, where $K=8, M_{\mathrm{RF}}=18$, $M=64, \rho_{\mathrm{BS}}=0.5$, and the direct AE selection scheme is considered.

$M=64$ approaches the BER achieved for transmission over uncor- 399 related Rayleigh-fading MIMO channels, which confirms the near- 400 optimal performance of the direct AE selection scheme.

Fig. 3 compares the overall BER achieved by the CS-based signal 402 detector and by the proposed SCS-based MUD versus the SNR in our 403 LS-SM-MIMO context, where $K=8, M_{\mathrm{RF}}=18, M=64, \rho_{\mathrm{BS}}=404$ 0.5 , and the direct AE selection scheme is considered. The SCS-based 405 MUD outperforms the CS-based signal detector even for $J=1$, since 406 the distributed sparsity of the aggregate SM signal is exploited. For the 407 SCS-based MUD, the BER performance improves when $J$ increases, 408 albeit this is achieved at the cost of reduced UL throughput. To mitigate 409 this impediment, a higher number of AEs can be employed by the users 410 for expanding the spatial constellation symbol set constituted by the 411 AEs. Specifically, by increasing $n_{t}$ from 4 to 8, the UL throughput 412 of the SCS-based MUD may be increased, but having more AEs at 413 the user results in a higher $\rho_{\mathrm{US}}$. When $n_{t}$ is increased, the BER 414 performance of the SCS-based MUD associated with $J=1$ degrades, 415 as expected. By contrast, when $n_{t}$ is increased, the BER performance 416 loss of the SCS-based MUD using $J=2$ can be less than $0.2 \mathrm{~dB}$ if the 417 BER of $10^{-4}$ is considered, even when a higher $\rho_{\mathrm{US}}$ associated with a 418 higher $n_{t}$ is considered.

419

Fig. 4 portrays the BER achieved by the different signal detectors as 420 a function of the SNR in the context of the proposed LS-SM-MIMO 421 for $K=8, M_{\mathrm{RF}}=18, M=64, n_{t}=4, \rho_{\mathrm{BS}}=0.5$, and $\rho_{\mathrm{US}}=0,422$ where the direct $\mathrm{AE}$ selection scheme is also considered. In Fig. 4, 423 we also characterize the 'oracle-assisted' LS-based signal detector 424 relying on the assumption that the spatial constellation symbol is 425 perfectly known at the BS for the proposed LS-SM-MIMO scheme 426 associated with $J=2,64-\mathrm{QAM}$ as well as for the MMSE-based 427 LS-MIMO detector in conjunction with 64-QAM, where both of them 428 only consider the BER of the classic signal constellation symbol. Here, 429 we assume that the LS-MIMO arrangement uses the same number 430 of RF chains to serve eight single-antenna users communicating 431 over uncorrelated Rayleigh-fading channels. The superiority of our 432 SCS-based MUD over the MMSE- and CS-based signal detectors 433 becomes clear.

Moreover, the performance gap between the oracle LS-based signal 435 detector associated with $7 \mathrm{bpcu}$ and the proposed SCS-based MUD 436 with 7 bpcu is less than $0.5 \mathrm{~dB}$. Note again that the oracle LS-based sig- 437 nal detector only considers the BER of the classic signal constellation 438 


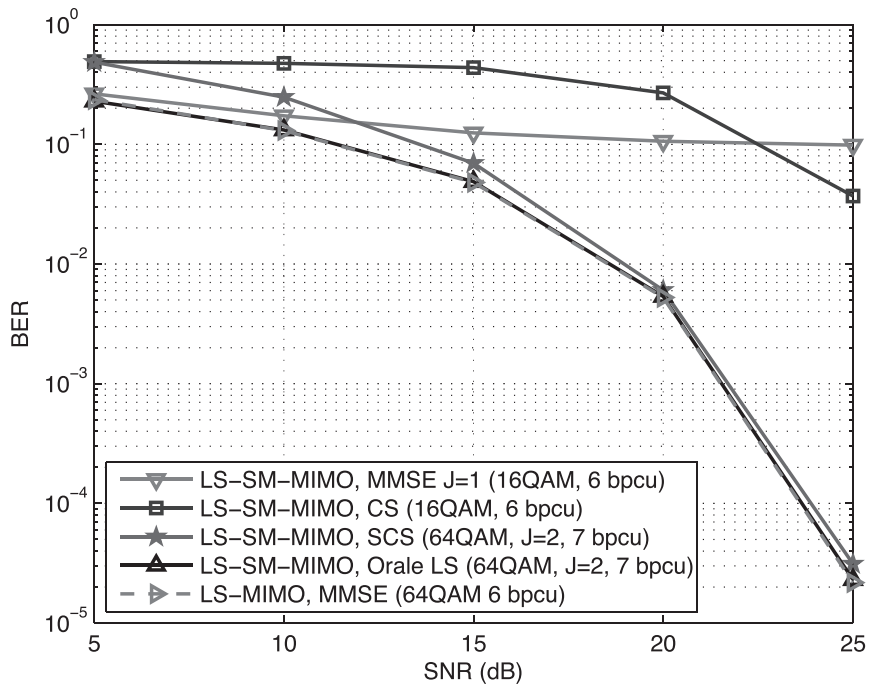

Fig. 4. Total BERs achieved by different signal detectors against different SNRs in the proposed LS-SM-MIMO and conventional LS-MIMO.

439 symbol, whereas the proposed SCS-based MUD considers both the 440 spatial and the classic signal constellation symbols. Finally, compared 441 with the conventional LS-MIMO using the MMSE-based signal de442 tector (6 bpcu), our proposed UL LS-SM-MIMO and the associated 443 SCS-based MUD (7 bpcu) only suffer from a negligible BER loss, 444 which explicitly confirmed the improved UL throughput of the pro445 posed LS-SM-MIMO scheme.

\section{V. CONCLUSION}

447 We have proposed an LS-SM-MIMO scheme for the UL transmis448 sion. The BS employs a large number of AEs but a much smaller 449 number of RF chains, where a simple receive AE selection scheme is 450 used for the improved performance. Each user equipped with multiple 451 AEs but only a single RF chain uses CPSC to combat multipath chan452 nels. SM has been adopted for the UL transmission to improve the UL 453 throughput. The proposed scheme is particularly suitable for scenarios 454 where a large number of low-cost AEs can be accommodated, and 455 both power consumption and hardware cost are heavily determined 456 by the number of RF chains. Due to the reduced number of RF chains 457 at the BS and multiple AEs employed by each user, the UL multiuser 458 signal detection is a challenging large-scale underdetermined problem. 459 We have proposed a joint SM transmission scheme at the users to 460 introduce the group sparsity of multiple aggregate SM signals, and a matching SCS-based MUD at the BS has been proposed to leverage the 461 inherently distributed sparsity of the aggregate SM signal as well as the 462 group sparsity of multiple aggregate SM signals for reliable multiuser 463 signal detection performance. The proposed SCS-based MUD enjoys 464 the low complexity, and our simulation results have demonstrated that 465 it performs better than its conventional counterparts with even much 466 higher UL throughput.

\section{REFERENCES}

468

[1] M. D. Renzo, H. Haas, A. Ghrayeb, S. Sugiura, and L. Hanzo, "Spatial 469 modulation for generalized MIMO: Challenges, opportunities and 470 implementation," Proc. IEEE, vol. 102, no. 1, pp. 56-103, Jan. 2014.471

[2] A. Younis, R. Mesleh, M. Di Renzo, and H. Haas, "Generalised spatial 472 modulation for large-scale MIMO," in Proc. EUSIPCO, Sep. 2014, 473 pp. 346-350.

[3] S. Ganesan, R. Mesleh, H. Haas, C. Ahn, and S. Yun, "On the performance 475 of spatial modulation OFDM," in Proc. 40th Asilomar Conf. Signals, Syst. 476 Comput., Oct. 2006, pp. 1825-1829.

[4] F. Rusek et al. "Scaling up MIMO: Opportunities and challenges with 478 very large arrays," IEEE Signal Process. Mag., vol. 30, no. 1, pp. 40-60, 479 Jan. 2013.

[5] N. Serafimovski et al., "Practical implementation of spatial modulation," 481 IEEE Trans. Veh. Technol., vol. 62, no. 9, pp. 4511-4523, Nov. 2013.

[6] P. Som and A. Chockalingam, "Spatial modulation and space shift 483 keying in single carrier" in Proc. IEEE Int. Symp. PIMRC, Sep. 2012, 484 pp. 1062-1067.

[7] X. Wu, M. Di Renzo, and H. Haas, "Adaptive selection of antennas for 486 optimum transmission in spatial modulation," IEEE Trans. Wireless 487 Commun., vol. 14, no. 7, pp. 3630-3641, Jul. 2015.

[8] S. Narayanan et al., "Multi-user spatial modulation MIMO" in Proc. IEEE 489 WCNC, Apr. 2014, pp. 671-676.

[9] X. Wu, H. Claussen, M. D. Renzo, and H. Haas, "Channel estimation 491 for spatial modulation," IEEE Trans. Commun., vol. 62, no. 12, 492 pp. 4362-4372, Dec. 2014

[10] A. Younis, S. Sinanovic, M. Di Renzo, R. Mesleh, and H. Haas, 494 "Generalised sphere decoding for spatial modulation," IEEE Trans. 495 Commun., vol. 61, no. 7, pp. 2805-2815, Jul. 2013.

[11] W. Liu, N. Wang, M. Jin, and H. Xu, "Denoising detection for the gener- 497 alized spatial modulation system using sparse property," IEEE Commun. 498 Lett., vol. 18, no. 1, pp. 22-25, Jan. 2014.

Cection with integer constraint 50 using multipath matching pursuit," IEEE Commun. Lett., vol. 18, no. 10, 501 pp. 1851-1854, Oct. 2014

[13] C. Yu et al., "Compressed sensing detector design for space shift keying 503 in MIMO systems," IEEE Commun. Lett., vol. 16, no. 10, pp. 1556-1559, 504 Oct. 2012.

[14] M. F. Duarte and Y. C. Eldar, "Structured compressed sensing: From 506 theory to applications," IEEE Trans. Signal Process., vol. 59, no. 9, 507 pp. 4053-4085, Sep. 2011.

[15] W. Dai and O. Milenkovic, "Subspace pursuit for compressive sens- 509 ing signal reconstruction," IEEE Trans. Inf. Theory, vol. 55, no. 5, 510 pp. 2230-2249, May 2009.

[16] A. Björck, Numerical Methods for Matrix Computations. Cham, 512 Switzerland: Springer Int. Publ. AG, 2014. 


\section{AUTHOR QUERY}

NO QUERY. 